Supporting Information

\title{
Photoreforming of non-recyclable plastic waste over a carbon nitride/nickel phosphide catalyst
}

Taylor Uekert, Hatice Kasap, Erwin Reisner*

Christian Doppler Laboratory for Sustainable SynGas Chemistry, Department of Chemistry, University of Cambridge, Lensfield Road, Cambridge CB2 1EW, UK. *e-mail: reisner@ch.cam.ac.uk 


\section{List of abbreviations}

$\mathrm{CN}_{\mathrm{x}}$ - cyanamide-functionalized carbon nitride

${ }_{2}{ }^{\mathrm{N}} \mathrm{CN}_{\mathrm{x}}$ - unfunctionalized carbon nitride

$E G$ - ethylene glycol

LA - lactic acid

$\mathrm{Ni}_{2} \mathrm{P}$ - nickel phosphide

PE - polyethylene

PET - polyethylene terephthalate

PLA - polylactic acid

PP - polypropylene

PR - photoreforming

PS - polystyrene

PUR - polyurethane

Rubber - polystyrene-block-polybutadiene

\section{Thermodynamic calculations}

Gibbs free energies were obtained or calculated from data in the cited references:

PET hydrolysis: $\mathrm{C}_{10} \mathrm{H}_{8} \mathrm{O}_{4}+2 \mathrm{H}_{2} \mathrm{O} \stackrel{K O H}{\longrightarrow} \mathrm{C}_{2} \mathrm{H}_{6} \mathrm{O}_{2}+\mathrm{C}_{8} \mathrm{H}_{6} \mathrm{O}_{4}, \Delta \mathrm{G}^{\circ}=66 \mathrm{~kJ} \mathrm{~mol}^{-1}$

PR of $E G: \mathrm{C}_{2} \mathrm{H}_{6} \mathrm{O}_{2}+2 \mathrm{H}_{2} \mathrm{O} \stackrel{h v, C N x}{\longrightarrow} 5 \mathrm{H}_{2}+2 \mathrm{CO}_{2}, \Delta \mathrm{G}^{\circ}=9.2 \mathrm{~kJ} \mathrm{~mol}^{-1}, \mathrm{E}_{\text {cell }}^{\circ}=-0.01 \mathrm{~V}[\mathrm{~S} 2]^{2}$

PLA hydrolysis: $\mathrm{C}_{3} \mathrm{H}_{4} \mathrm{O}_{2}+\mathrm{H}_{2} \mathrm{O} \stackrel{K O H}{\longrightarrow} \mathrm{C}_{3} \mathrm{H}_{6} \mathrm{O}_{3}, \Delta \mathrm{G}^{\circ}=82 \mathrm{~kJ} \mathrm{~mol}^{-1}$

PR of $L A: \mathrm{C}_{3} \mathrm{H}_{6} \mathrm{O}_{3}+3 \mathrm{H}_{2} \mathrm{O} \stackrel{h v, C N x}{\longrightarrow} 6 \mathrm{H}_{2}+3 \mathrm{CO}_{2}, \Delta \mathrm{G}^{\circ}=27 \mathrm{~kJ} \mathrm{~mol}^{-1}, \mathrm{E}_{\text {cell }}^{\circ}=-0.02 \mathrm{~V}$ 


\section{Supplementary Tables}

Table S1. Inductively coupled plasma optical emission spectrometry (ICP-OES) quantification of $\mathrm{Ni}$ and $\mathrm{P}$ content of several catalysts. Samples (typically $\sim 3 \mathrm{mg}$ ) were dissolved in $2 \mathrm{~mL}$ of $2: 1 \mathrm{H}_{2} \mathrm{O}_{2}: \mathrm{H}_{2} \mathrm{SO}_{4}$ overnight, diluted with $\mathrm{H}_{2} \mathrm{O}$ and then submitted for measurement.

\begin{tabular}{|c|c|c|c|c|}
\hline Catalyst & $\begin{array}{l}\text { Ideal Ni content } \\
\left(\mathrm{mg}_{\mathrm{Ni}} \mathrm{g}_{\mathrm{CNx}}{ }^{-1}\right)\end{array}$ & $\begin{array}{c}\text { Measured } \mathrm{Ni} \\
\text { content } \\
\left(\mathrm{mg}_{\mathrm{Ni}} \mathrm{g}_{\mathrm{cNx}}{ }^{-1}\right)\end{array}$ & $\begin{array}{l}\text { Ideal P content } \\
\left(\mathrm{mg}_{\mathrm{P}} \mathrm{gCNx}^{-1}\right)\end{array}$ & $\begin{array}{c}\text { Measured } P \\
\text { content } \\
\left(\mathrm{mgP}_{\mathrm{CNn}}{ }^{-1}\right)\end{array}$ \\
\hline $\mathrm{CN}_{\mathrm{x}} \mid \mathrm{Ni}_{2} \mathrm{P}$ & 15.9 & 15.3 & 4.2 & 52.2 \\
\hline $\mathrm{CN}_{x} \mid \mathrm{Ni}_{2} \mathrm{P}$ post-PR & 15.9 & 15.1 & 4.2 & 8.8 \\
\hline solution post-PR[a] & 0.00 & 0.14 & n.m. & n.m. \\
\hline $\mathrm{CN}_{\mathrm{x}}-\mathrm{P}^{[\mathrm{b}]}$ & -- & -- & 5.0 & 40.3 \\
\hline $\mathrm{CN}_{\mathrm{x}}-\mathrm{PO}_{\mathrm{x}}[\mathrm{c}]$ & -- & -- & 4.9 & 35.4 \\
\hline$\left.{ }^{H} 2 \mathrm{~N} C \mathrm{~N}_{x}-\mathrm{P}-\mathrm{b}\right]$ & -- & -- & 5.0 & 14.0 \\
\hline
\end{tabular}

n.m. $=$ not measured

[a] The photocatalyst was removed via centrifugation, and only the supernatant was submitted for ICP analysis.

[b] $\mathrm{CN}_{\mathrm{x}}-\mathrm{P}$ and ${ }^{\mathrm{H} 2 \mathrm{~N}} \mathrm{CN}_{\mathrm{x}}-\mathrm{P}$ were synthesized according to the $\mathrm{CN}_{\mathrm{x}} \mid \mathrm{Ni}_{2} \mathrm{P}$ procedure, but without the addition of the Ni precursor.

[c] $\mathrm{CN}_{x}-\mathrm{PO}_{x}$ was prepared by replacing $\mathrm{NaH}_{2} \mathrm{PO}_{2} \cdot \mathrm{H}_{2} \mathrm{O}$ with $\mathrm{Na}_{3} \mathrm{PO}_{4}$ in the above synthesis. 
Table S2. X-ray photoelectron spectroscopy (XPS) survey quantification of $\mathrm{CN}_{x}, \mathrm{Ni}_{2} \mathrm{P}, \mathrm{CN}_{\mathrm{x}} \mid \mathrm{Ni}_{2} \mathrm{P}(2$ wt\%), post-catalysis $\mathrm{CN}_{x} \mid \mathrm{Ni}_{2} \mathrm{P}(2 \mathrm{wt} \%)$, and $\mathrm{CN}_{x} \mid \mathrm{P}$. All powders were dispersed in ethanol and dropcast on FTO glass slides prior to characterization.

\begin{tabular}{|c|c|c|c|c|c|c|}
\hline \multirow[b]{2}{*}{ Sample } & \multicolumn{6}{|c|}{ Atomic concentration (\%) } \\
\hline & O 1s & C 1s & $\mathrm{N} 1 \mathrm{~s}$ & $\mathrm{~K} 2 \mathrm{~s}$ & $\mathrm{Ni} 2 \mathrm{p}_{3 / 2}$ & $P 2 p$ \\
\hline $\mathrm{CN}_{\mathrm{x}}$ area 1 & 12.54 & 34.33 & 41.38 & 5.89 & & \\
\hline $\mathrm{CN}_{\mathrm{x}}$ area 2 & 23.37 & 27.85 & 30.89 & 5.08 & $\overline{--}$ & $\overline{--}$ \\
\hline $\mathrm{CN}_{\mathrm{x}}$ area 3 & 17.75 & 33.63 & 34.79 & 5.24 & -- & -- \\
\hline Average & 17.89 & 31.94 & 35.69 & 5.40 & -- & -- \\
\hline $\mathrm{Ni}_{2} \mathrm{P}$ area 1 & 47.82 & 20.21 & -- & -- & 4.67 & 17.90 \\
\hline $\mathrm{Ni}_{2} \mathrm{P}$ area 2 & 41.26 & 30.02 & -- & -- & 4.18 & 16.74 \\
\hline $\mathrm{Ni}_{2} \mathrm{P}$ area 3 & 46.59 & 20.55 & -- & -- & 5.50 & 21.50 \\
\hline Average & 45.22 & 23.59 & -- & -- & 4.78 & 18.71 \\
\hline $\mathrm{CN}_{\times} \mid \mathrm{Ni}_{2} \mathrm{P}$ area 1 & 13.09 & 35.18 & 39.17 & 3.80 & 0.37 & 1.93 \\
\hline $\mathrm{CN}_{\times} \mid \mathrm{Ni}_{2} \mathrm{P}$ area 2 & 18.76 & 31.46 & 34.90 & 3.94 & 0.25 & 1.38 \\
\hline $\mathrm{CN}_{\mathbf{x}} \mid \mathrm{Ni}_{2} \mathrm{P}$ area 3 & 14.73 & 33.74 & 38.48 & 4.01 & 0.30 & 1.75 \\
\hline Average & 15.53 & 33.46 & 37.52 & 3.92 & 0.31 & 1.69 \\
\hline Post-PR $\mathrm{CN}_{\mathrm{x}} \mid \mathrm{Ni}_{2} \mathrm{P}$ area 1 & 29.07 & 26.06 & 24.81 & 1.15 & 0.19 & -- \\
\hline Post-PR $\mathrm{CN}_{\mathrm{x}} \mid \mathrm{Ni}_{2} \mathrm{P}$ area 2 & 27.65 & 27.36 & 25.90 & 1.84 & 0.18 & -- \\
\hline Post-PR $\mathrm{CN}_{\mathrm{x}} \mid \mathrm{Ni}_{2} \mathrm{P}$ area 3 & 23.95 & 29.01 & 29.89 & 1.62 & 0.28 & -- \\
\hline Average & 26.89 & 27.48 & 26.87 & 1.54 & 0.22 & -- \\
\hline $\mathrm{CN}_{\mathrm{x}} \mid \mathrm{P}$ area 1 & 7.59 & 40.66 & 42.76 & 3.91 & -- & 1.54 \\
\hline $\mathrm{CN}_{\mathrm{x}} \mid \mathrm{P}$ area 2 & 6.71 & 41.10 & 43.48 & 4.20 & -- & 1.88 \\
\hline Average & 7.15 & 40.88 & 43.12 & 4.05 & -- & 1.71 \\
\hline
\end{tabular}

Table S3. Comparison of the synthesized $\mathrm{CN}_{x} \mid \mathrm{Ni}_{2} \mathrm{P}$ catalyst to reported ${ }^{\mathrm{H} 2 \mathrm{~N}} \mathrm{CN}_{x} \mid \mathrm{Ni}_{2} \mathrm{P}$ catalysts for $\mathrm{H}_{2}$ evolution with triethanolamine as hole scavenger. All cited samples were irradiated with $300 \mathrm{~W} \mathrm{Xe}$ lamps with $\lambda>420 \mathrm{~nm}$ cutoff filters (no temperatures cited). Samples labelled as "this work" were irradiated with a $1000 \mathrm{~W}$ Xe lamp (AM $1.5 \mathrm{G}, 100 \mathrm{~mW} \mathrm{~cm}^{-2}, 25^{\circ} \mathrm{C}$ ) with a $\lambda>420 \mathrm{~nm}$ cutoff filter.

\begin{tabular}{|c|c|c|c|c|c|c|c|}
\hline Catalyst & $\begin{array}{l}\text { [Catalyst] } \\
\left(\mathrm{mg} \mathrm{mL}^{-1}\right)\end{array}$ & $\begin{array}{l}\text { [Substrate] } \\
\left(\mathrm{mg} \mathrm{mL}^{-1}\right)\end{array}$ & $\begin{array}{c}\text { Reactor } \\
\text { Volume } \\
\text { (mL) }\end{array}$ & $\begin{array}{l}\text { Time } \\
\text { (h) }\end{array}$ & $\begin{array}{c}\text { Yield } \pm \sigma \\
\left(\mu \text { mol }_{H_{2}} \mathrm{~g}_{\text {sub }^{-1}}\right)\end{array}$ & $\begin{array}{c}\text { Activity } \pm \sigma \\
\left(\mu \mathrm{mol}_{\mathrm{H}_{2}} \mathrm{~g}_{\left.\text {cat }^{-1} \mathrm{~h}^{-1}\right)}\right.\end{array}$ & Ref \\
\hline $\mathrm{CN}_{x} \mid \mathrm{Ni}_{2} \mathrm{P} 2 \%{ }^{[\mathrm{a}]}$ & 1.2 & 113 & 7.91 & 20 & $33.4 \pm 1.7$ & $118 \pm 6.0$ & * \\
\hline${ }^{\mathrm{H}_{2}} \mathrm{~N} \mathrm{CN}_{x} \mid \mathrm{Ni}_{2} \mathrm{P} 2 \%$ & 0.83 & 113 & 60 & 20 & 29.5 & 200 & 5 \\
\hline $\mathrm{H}_{2} \mathrm{~N} \mathrm{CN}_{x} \mid \mathrm{Ni}_{2} \mathrm{P} 2 \%$ & 1.2 & 113 & 7.91 & 20 & $27.4 \pm 1.4$ & $96.7 \pm 4.9$ & * \\
\hline $\mathrm{H}_{2} \mathrm{~N} \mathrm{CN}_{\mathrm{x}} \mid \mathrm{Ni}_{2} \mathrm{P} 3 \%$ & 1.0 & 226 & 100 & 4 & 26.6 & 1503 & 6 \\
\hline $\mathrm{H}_{2} \mathrm{~N} \mathrm{CN}_{x} \mathrm{Ni}_{12} \mathrm{P}_{5} 2 \%$ & 1.0 & 113 & 80 & 20 & 14.6 & 82.5 & 7 \\
\hline $\mathrm{H}_{2} \mathrm{~N}_{\mathrm{CN}} \mid \mathrm{Ni}_{2} \mathrm{P} 3.5 \%$ & 0.45 & 103 & 250 & 4 & 8.39 & 474 & 8 \\
\hline $\mathrm{H}_{2} \mathrm{~N} \mathrm{~N}_{x} \mid \mathrm{Ni}_{2} \mathrm{P} \quad 0.48 \%$ & 1.0 & 113 & 37 & 2 & 10.2 & 575 & 9 \\
\hline${ }^{\mathrm{H}_{2}} \mathrm{~N} \mathrm{CN}_{x} \mid \mathrm{Ni}_{2} \mathrm{P} 2 \%$ & 0.5 & 170 & 100 & 3 & 1.12 & 127 & 10 \\
\hline
\end{tabular}

[a] Percentages indicate wt\%.

* This work. 
Table S4. Optimisation of photoreforming conditions with $\mathrm{CN}_{x} \mid \mathrm{Ni}_{2} \mathrm{P}$. Conditions (unless stated otherwise below): ultra-sonicated $\mathrm{CN}_{x} \mid \mathrm{Ni}_{2} \mathrm{P} 2 \mathrm{wt} \%$ (3.2 mg), pre-treated PET (50 mg), aqueous $\mathrm{KOH}(1 \mathrm{M}, 2 \mathrm{~mL}$ ), sealed photoreactor (internal volume $7.91 \mathrm{~mL}$ ) under anaerobic conditions, simulated solar light $(20 \mathrm{~h}$ AM $1.5 \mathrm{G}, 100 \mathrm{~mW} \mathrm{~cm}^{-2}, 25^{\circ} \mathrm{C}$ ). Yields and activities are cumulative values. $\sigma$ is the standard deviation calculated from 3 samples, unless stated otherwise.

\begin{tabular}{|c|c|c|c|}
\hline Description & $\begin{array}{l}\mathrm{Ni}_{2} \mathrm{P} \text { loading } \\
\text { (wt \%) }\end{array}$ & $\begin{array}{c}\text { Yield } \pm \sigma \\
\left(\mu \mathrm{mol}_{2} \mathrm{~g}_{\mathrm{sub}^{-1}}\right)^{-1}\end{array}$ & $\begin{array}{c}\text { Activity } \pm \sigma \\
\left(\mu \mathrm{mol}_{2} \mathrm{gcat}^{-1} \mathbf{h}^{-1}\right)\end{array}$ \\
\hline $\begin{array}{l}\mathrm{Ni}_{2} P \text { concentration } \\
\text { optimisation }\end{array}$ & $\begin{array}{c}0.5 \\
2 \\
5\end{array}$ & $\begin{array}{c}2.11 \pm 0.13 \\
27.6 \pm 3.4 \\
29.4 \pm 1.5\end{array}$ & $\begin{array}{c}1.59 \pm 0.10 \\
21.6 \pm 2.7 \\
23.0 \pm 1.2\end{array}$ \\
\hline Description & $\begin{array}{l}{\left[\mathrm{CN}_{\mathbf{x}} \mid \mathrm{Ni}_{2} \mathrm{P}\right]} \\
\left(\mathrm{mg} \mathrm{mL}^{-1}\right)\end{array}$ & $\begin{array}{c}\text { Yield } \\
\left(\mu \mathrm{mol}_{\mathrm{H}_{2}} \mathrm{~g}_{\mathrm{sub}^{-1}}\right)^{-1}\end{array}$ & $\begin{array}{c}\text { Activity } \\
\left(\mu \mathrm{mol}_{\mathrm{H}_{2}} \mathrm{gcat}^{-1} \mathrm{~h}^{-1}\right)\end{array}$ \\
\hline $\begin{array}{c}C N_{x} \text { concentration } \\
\text { optimisation }\end{array}$ & $\begin{array}{c}0.2^{[\mathrm{a}]} \\
0.5^{[\mathrm{a}]} \\
1^{[\mathrm{a}]} \\
1.6 \\
2^{[\mathrm{a}]}\end{array}$ & $\begin{array}{c}0.980 \pm 0.300 \\
1.45 \pm 0.25 \\
7.30 \pm 0.36 \\
27.6 \pm 3.4 \\
22.1 \pm 1.1\end{array}$ & $\begin{array}{c}6.12 \pm 1.87 \\
3.62 \pm 0.62 \\
9.12 \pm 0.46 \\
21.6 \pm 2.7 \\
13.8 \pm 0.7\end{array}$ \\
\hline Description & $\begin{array}{c}{[\mathrm{KOH}]} \\
(\mathrm{M})\end{array}$ & $\begin{array}{c}\text { Yield } \\
\left(\mathrm{mmol}_{\mathrm{H}_{2}} \mathrm{~g}_{\mathrm{sub}}{ }^{-1}\right)\end{array}$ & $\begin{array}{c}\text { Activity } \\
\left(\mu \mathrm{mol}_{2} \mathrm{gcat}^{-1} \mathrm{~h}^{-1}\right)\end{array}$ \\
\hline $\begin{array}{c}K O H \text { concentration } \\
\text { optimisation }\end{array}$ & $\begin{array}{c}0 \\
0.5 \\
1 \\
5 \\
10\end{array}$ & $\begin{array}{c}0.0 \pm 0.0 \\
0.800 \pm 0.007 \\
27.6 \pm 3.4 \\
83.8 \pm 5.3 \\
111 \pm 8\end{array}$ & $\begin{array}{c}0.0 \pm 0.0 \\
0.625 \pm 0.005 \\
21.6 \pm 2.7 \\
65.4 \pm 2.2 \\
86.5 \pm 6.2\end{array}$ \\
\hline
\end{tabular}

[a] $\sigma$ calculated from 2 samples.

Table S5. Comparison of photoreforming with pre-treated versus non-treated PET. Conditions: ultrasonicated $\mathrm{CN}_{\mathrm{x}} \mid \mathrm{Ni}_{2} \mathrm{P} 2 \mathrm{wt} \%$ (3.2 mg), PET (50 mg), aq. $\mathrm{KOH}(1 \mathrm{M}, 2 \mathrm{~mL}$ ), sealed photoreactor (internal volume $7.91 \mathrm{~mL}$ ) under anaerobic conditions, simulated solar light (AM 1.5G, $100 \mathrm{~mW} \mathrm{~cm}{ }^{-2}, 25{ }^{\circ} \mathrm{C}$ ). Yields and activities are cumulative values. $\sigma$ is the standard deviation calculated from 3 samples.

\begin{tabular}{|c|c|c|c|}
\hline Description & $\begin{array}{c}\text { Time } \\
\text { (h) }\end{array}$ & $\begin{array}{c}\text { Yield } \pm \sigma \\
\left(\mu \mathrm{mol}_{H_{2}} \mathrm{~g}_{\text {sub }^{-1}}\right)\end{array}$ & $\begin{array}{c}\text { Activity } \pm \sigma \\
\left(\mu \mathrm{mol}_{H_{2}} \mathrm{gcat}^{-1} \mathrm{~h}^{-1}\right)\end{array}$ \\
\hline \multirow{3}{*}{ No pre-treatment } & 4 & $5.00 \pm 0.34$ & $19.5 \pm 1.3$ \\
\hline & 20 & $17.1 \pm 0.9$ & $12.8 \pm 0.6$ \\
\hline & 22 & $18.8 \pm 1.7$ & $12.8 \pm 1.1$ \\
\hline \multirow{3}{*}{ With pre-treatment } & 4 & $5.06 \pm 0.34$ & $19.8 \pm 1.3$ \\
\hline & 20 & $27.6 \pm 3.4$ & $21.6 \pm 2.7$ \\
\hline & 22 & $30.6 \pm 6.1$ & $21.7 \pm 4.3$ \\
\hline
\end{tabular}


Table S6. Quantification (by ${ }^{1} \mathrm{H}$-NMR spectroscopy) of polymer solubilization after pre-treatment (before photocatalysis).

\begin{tabular}{ccccc}
\hline Sample & Components & $\begin{array}{c}\text { Ideal quantity of } \\
\text { component } \mathbf{( m g )}\end{array}$ & $\begin{array}{c}\text { Measured } \\
\text { quantity of } \\
\text { component }(\mathbf{m g})\end{array}$ & \% solubilization \\
\hline \multirow{2}{*}{ PET } & ethylene glycol & 2.1 & 1.3 & 62 \\
& terephthalate & 5.7 & 2.9 & 51 \\
\hline \multirow{2}{*}{ PET bottle } & lactate & 6.0 & 4.3 & 72 \\
& ethylene glycol & 1.2 & 0.7 & 48 \\
\hline \multirow{2}{*}{ Polyester fiber } & terephthalate & 3.1 & 1.3 & 25 \\
& ethylene glycol & 1.2 & 0.3 & 26 \\
\hline
\end{tabular}

Table S7. Comparison of photoreforming with ultra-sonicated versus un-sonicated $\mathrm{CN}_{\mathrm{x}} \mid \mathrm{Ni}_{2} \mathrm{P}$. Conditions: $\mathrm{CN}_{x} \mid \mathrm{Ni}_{2} \mathrm{P} 2 \mathrm{wt} \%(3.2 \mathrm{mg})$, pre-treated PET $(50 \mathrm{mg})$, aq. $\mathrm{KOH}(1 \mathrm{M}, 2 \mathrm{~mL})$, sealed photoreactor (internal volume $7.91 \mathrm{~mL}$ ) under anaerobic conditions, simulated solar light (AM 1.5G, 100 $\mathrm{mW} \mathrm{cm}{ }^{-2}, 25^{\circ} \mathrm{C}$ ). Yields and activities are cumulative values. $\sigma$ is the standard deviation calculated from 3 samples.

\begin{tabular}{|c|c|c|c|}
\hline Description & $\begin{array}{c}\text { Time } \\
\text { (h) }\end{array}$ & 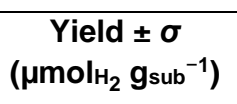 & $\begin{array}{c}\text { Activity } \pm \sigma \\
\left(\mu_{\mathrm{mol}} \mathrm{H}_{2} \mathrm{~g}_{\left.\mathrm{cat}^{-1} \mathrm{~h}^{-1}\right)}\right.\end{array}$ \\
\hline \multirow{2}{*}{ No sonication } & 4 & $3.86 \pm 0.19$ & $15.1 \pm 0.7$ \\
\hline & 20 & $6.34 \pm 0.44$ & $4.95 \pm 0.35$ \\
\hline \multirow{2}{*}{ With ultra-sonication } & 4 & $5.06 \pm 0.34$ & $19.8 \pm 1.3$ \\
\hline & 20 & $27.6 \pm 3.4$ & $21.6 \pm 2.7$ \\
\hline
\end{tabular}


Table S8. Control experiments for photoreforming of polymers over $\mathrm{CN}_{x} \mid \mathrm{Ni}_{2} P$. Conditions (unless stated otherwise below): ultra-sonicated $\mathrm{CN}_{x} \mid \mathrm{Ni}_{2} \mathrm{P} 2 \mathrm{wt} \%$ (3.2 mg), pre-treated polymer (50 mg), aqueous $\mathrm{KOH}$ (1 M, $2 \mathrm{~mL}$ ), sealed photoreactor (internal volume $7.91 \mathrm{~mL}$ ) under anaerobic conditions, simulated solar light (AM 1.5G, $100 \mathrm{~mW} \mathrm{~cm}^{-2}, 25^{\circ} \mathrm{C}$ ). Yields and activities are cumulative values. $\sigma$ is the standard deviation calculated from 3 samples.

\begin{tabular}{|c|c|c|c|c|}
\hline Description & & $\begin{array}{l}\text { Time } \\
\text { (h) }\end{array}$ & $\begin{array}{l}\text { Yield } \pm \sigma \\
\left(\mu \mathrm{mol}_{\mathrm{H}_{2}}\right)\end{array}$ & $\begin{array}{c}\text { Activity } \\
\left(\mu \mathrm{mol}_{H_{2}} \mathrm{~g}_{\mathrm{cat}^{-1}} \mathrm{~h}^{-1}\right)\end{array}$ \\
\hline \multirow{13}{*}{ No substrate $e^{[a, b]}$} & & 2 & $0.049 \pm 0.021$ & $7.66 \pm 3.28$ \\
\hline & & 4 & $0.053 \pm 0.044$ & $4.14 \pm 3.44$ \\
\hline & & 20 & $0.132 \pm 0.065$ & $2.06 \pm 1.01$ \\
\hline & & 25 & $0.134 \pm 0.011$ & $1.67 \pm 0.14$ \\
\hline & & 27 & $0.145 \pm 0.013$ & $1.68 \pm 0.15$ \\
\hline & & 44 & $0.171 \pm 0.016$ & $1.21 \pm 0.11$ \\
\hline & & 46 & $0.175 \pm 0.016$ & $1.19 \pm 0.11$ \\
\hline & & 50 & $0.180 \pm 0.017$ & $1.12 \pm 0.11$ \\
\hline & & 24 & $0.071 \pm 0.003$ & $0.924 \pm 0.046$ \\
\hline & & 48 & $0.074 \pm 0.011$ & $0.482 \pm 0.072$ \\
\hline & & 72 & $0.140 \pm 0.010$ & $0.608 \pm 0.043$ \\
\hline & & 96 & $0.208 \pm 0.023$ & $0.677 \pm 0.075$ \\
\hline & & 120 & $0.269 \pm 0.059$ & $0.700 \pm 0.153$ \\
\hline Description & Substrate & $\begin{array}{l}\text { Time } \\
\text { (h) }\end{array}$ & $\begin{array}{c}\text { Yield } \\
\left(\mu \mathrm{mol}_{\mathrm{H}_{2}} \mathrm{~g}_{\mathrm{sub}^{-1}}\right)\end{array}$ & $\begin{array}{c}\text { Activity } \\
\left(\mu \mathrm{mol}_{\mathrm{H}_{2}} \mathrm{~g}_{\mathrm{cat}}{ }^{-1} \mathrm{~h}^{-1}\right)\end{array}$ \\
\hline \multirow{3}{*}{ No light } & PET & 4 & $0.0 \pm 0.0$ & $0.0 \pm 0.0$ \\
\hline & PIA & 4 & $0.0 \pm 0.0$ & $0.0 \pm 0.0$ \\
\hline & & 20 & $0.0 \pm 0.0$ & $0.0 \pm 0.0$ \\
\hline \multirow{4}{*}{ No catalyst } & PET & 4 & $0.0 \pm 0.0$ & $0.0 \pm 0.0$ \\
\hline & & 20 & $0.0 \pm 0.0$ & $0.0 \pm 0.0$ \\
\hline & PLA & 4 & $0.0 \pm 0.0$ & $0.0 \pm 0.0$ \\
\hline & & 20 & $0.0 \pm 0.0$ & $0.0 \pm 0.0$ \\
\hline \multirow{4}{*}{$\begin{array}{c}\text { No co-catalyst } \\
\left(\mathrm{CN}_{x} \text { only }\right)\end{array}$} & PET & 4 & $0.0 \pm 0.0$ & $0.0 \pm 0.0$ \\
\hline & & 20 & $0.687 \pm 0.034$ & $0.537 \pm 0.027$ \\
\hline & PLA & 4 & $0.0 \pm 0.0$ & $0.0 \pm 0.0$ \\
\hline & & 20 & $1.24 \pm 0.22$ & $0.969 \pm 0.172$ \\
\hline \multirow{4}{*}{$\begin{array}{c}\text { No light-absorber } \\
\text { (Ni2P only) }\end{array}$} & PET & 4 & $0.0 \pm 0.0$ & $0.0 \pm 0.0$ \\
\hline & & 20 & $0.0 \pm 0.0$ & $0.0 \pm 0.0$ \\
\hline & & 4 & $0.0 \pm 0.0$ & $0.0 \pm 0.0$ \\
\hline & PLA & 20 & $0.0 \pm 0.0$ & $0.0 \pm 0.0$ \\
\hline \multirow{4}{*}{$\begin{array}{l}\text { Irradiated with } \lambda> \\
\quad 420 \mathrm{~nm} \text { filter }\end{array}$} & PET & 4 & $3.96 \pm 0.54$ & $15.5 \pm 2.1$ \\
\hline & & 20 & $5.52 \pm 0.74$ & $4.31 \pm 0.58$ \\
\hline & & 4 & $4.76 \pm 0.24$ & $18.6 \pm 0.9$ \\
\hline & PLA & 20 & $10.9 \pm 0.7$ & $8.54 \pm 0.56$ \\
\hline
\end{tabular}

[a] Different samples were used for the 2-50 $\mathrm{h}$ and 24-120 $\mathrm{h}$ timescales.

[b] The high initial activity is likely due to remnant $\mathrm{NaH}_{2} \mathrm{PO}_{2}$ from $\mathrm{Ni}_{2} \mathrm{P}$ co-catalyst synthesis. 
Table S9. Screening of noble-metal-free co-catalysts with $\mathrm{CN}_{\mathrm{x}}$ for photoreforming of PET. Conditions: ultra-sonicated $\mathrm{CN}_{x}(3.2 \mathrm{mg}$ ), co-catalyst, pre-treated PET (50 mg), aq. $\mathrm{KOH}(1 \mathrm{M}, 2 \mathrm{~mL})$, sealed photoreactor (internal volume $7.91 \mathrm{~mL}$ ) under anaerobic conditions, simulated solar light (AM 1.5G, $100 \mathrm{~mW}$ $\mathrm{cm}^{-2}, 25^{\circ} \mathrm{C}$ ). Yields are cumulative values. $\sigma$ is the standard deviation calculated from 3 samples.

\begin{tabular}{|c|c|c|c|}
\hline Co-catalyst & $\begin{array}{l}\text { Time } \\
\text { (h) }\end{array}$ & $\begin{array}{c}\text { Yield } \pm \sigma \\
\left(\mu_{\mathrm{mol}_{2}} \mathrm{~g}_{\left.\text {sub }^{-1}\right)}\right.\end{array}$ & $\begin{array}{c}\text { Activity } \pm \sigma \\
\left(\mu \mathrm{mol}_{\mathrm{H}_{2}} \mathrm{~g}_{\mathrm{cat}}{ }^{-1} \mathrm{~h}^{-1}\right)\end{array}$ \\
\hline \multirow{2}{*}{$\mathrm{Ni}, 5 \mathrm{wt} \%\left(\mathrm{Ni}\left(\mathrm{NO}_{3}\right)_{2} \cdot 6 \mathrm{H}_{2} \mathrm{O}\right)^{[\mathrm{a}]}$} & 4 & $0.0 \pm 0.0$ & $0.0 \pm 0.0$ \\
\hline & 20 & $11.8 \pm 2.5$ & $9.19 \pm 1.96$ \\
\hline \multirow{2}{*}{$\mathrm{Ni}, 5 \mathrm{wt} \%\left(\mathrm{Ni}\left(\mathrm{BF}_{4}\right)_{2} \cdot 6 \mathrm{H}_{2} \mathrm{O}\right)^{[\mathrm{a}, \mathrm{b}]}$} & 4 & 0.00 & 0.00 \\
\hline & 20 & 9.34 & 7.30 \\
\hline \multirow{2}{*}{$\mathrm{Ni}, 2 \mathrm{wt} \%\left(\mathrm{Ni}\left(\mathrm{acac}_{2}\right)_{3}\right)^{[\mathrm{c}]}$} & 4 & $0.0 \pm 0.0$ & $0.0 \pm 0.0$ \\
\hline & 20 & $9.44 \pm 0.49$ & $7.37 \pm 0.38$ \\
\hline \multirow{2}{*}{$\mathrm{NiO}, 5$ wt\%[d] } & 4 & $0.0 \pm 0.0$ & $0.0 \pm 0.0$ \\
\hline & 20 & $0.0 \pm 0.0$ & $0.0 \pm 0.0$ \\
\hline \multirow{2}{*}{$\mathrm{NiO}$ NPs, 5 wt\%[d] } & 4 & $0.0 \pm 0.0$ & $0.0 \pm 0.0$ \\
\hline & 20 & $0.0 \pm 0.0$ & $0.0 \pm 0.0$ \\
\hline \multirow{2}{*}{$\mathrm{Ni}(\mathrm{OH})_{2}, 5 \mathrm{wt} \%{ }^{[\mathrm{e}]}$} & 4 & $0.0 \pm 0.0$ & $0.0 \pm 0.0$ \\
\hline & 20 & $16.5 \pm 2.3$ & $12.9 \pm 1.80$ \\
\hline \multirow{2}{*}{$\mathrm{Ni}_{2} \mathrm{P}, 2$ wt $\%$} & 4 & $5.06 \pm 0.34$ & $19.8 \pm 1.3$ \\
\hline & 20 & $27.6 \pm 3.4$ & $21.6 \pm 2.7$ \\
\hline \multirow{2}{*}{$\mathrm{Fe}, 5 \mathrm{wt} \%\left(\mathrm{Fe}\left(\mathrm{NO}_{3}\right)_{3} \cdot 9 \mathrm{H}_{2} \mathrm{O}\right)^{[\mathrm{a}]}$} & 4 & $0.0 \pm 0.0$ & $0.0 \pm 0.0$ \\
\hline & 20 & $0.0 \pm 0.0$ & $0.0 \pm 0.0$ \\
\hline \multirow{2}{*}{$\mathrm{Fe}, 5 \mathrm{wt} \%\left(\mathrm{Fe}\left(\mathrm{BF}_{4}\right)_{2} \cdot 6 \mathrm{H}_{2} \mathrm{O}\right)^{[a, b]}$} & 4 & 0.0 & 0.0 \\
\hline & 20 & 1.76 & 1.37 \\
\hline \multirow{2}{*}{$\mathrm{Fe}_{2} \mathrm{O}_{3}, 5 \mathrm{wt} \%[\mathrm{~d}]$} & 4 & $0.0 \pm 0.0$ & $0.0 \pm 0.0$ \\
\hline & 20 & $0.0 \pm 0.0$ & $0.0 \pm 0.0$ \\
\hline \multirow{2}{*}{$\mathrm{Fe}_{3} \mathrm{O}_{4} \mathrm{NPs}, 5$ wt\%[d] } & 4 & $0.0 \pm 0.0$ & $0.0 \pm 0.0$ \\
\hline & 20 & $0.933 \pm 0.144$ & $0.729 \pm 0.112$ \\
\hline \multirow{2}{*}{$F e_{x} P, 2 w t^{[a, f]}$} & 4 & 0.0 & 0.0 \\
\hline & 20 & 0.620 & 0.484 \\
\hline \multirow{2}{*}{ CuO NPs, 5 wt\%[d] } & 4 & $0.0 \pm 0.0$ & $0.0 \pm 0.0$ \\
\hline & 20 & $0.0 \pm 0.0$ & $0.0 \pm 0.0$ \\
\hline \multirow{2}{*}{ Co, 5 wt $\%\left(\mathrm{Co}\left(\mathrm{BF}_{4}\right)_{2} \cdot 6 \mathrm{H}_{2} \mathrm{O}\right)^{[a, b]}$} & 4 & 0.00 & 0.00 \\
\hline & 20 & 4.54 & 2.95 \\
\hline
\end{tabular}

[a] Metal salts were simply dissolved in $\mathrm{H}_{2} \mathrm{O}$ and added to $\mathrm{CN}_{x}$.

[b] Single run measurements.

${ }^{[c]} \mathrm{CN}_{x} \mid \mathrm{Ni}$ was synthesized as previously reported. ${ }^{5} \mathrm{Ni}(\mathrm{II})$ acetylacetone $(11 \mathrm{mg})$ and $\mathrm{CN}_{\mathrm{x}}(150 \mathrm{mg})$ were mixed in a minimum of water and stirred and sonicated for $1 \mathrm{~h}$ each. After drying under vacuum at 60 ${ }^{\circ} \mathrm{C}$, the powder was heated for $1 \mathrm{~h}$ at $200{ }^{\circ} \mathrm{C}$ under $\operatorname{Ar}\left(\right.$ ramp rate $\left.5{ }^{\circ} \mathrm{C} \mathrm{min}{ }^{-1}\right)$. The solid was cooled, washed with water $(3 x)$ and ethanol $(3 x)$, and dried under vacuum.

[d] Metal oxides were ground with $\mathrm{CN}_{x}$ with a pestle and mortar.

[e] A literature procedure was modified slightly. ${ }^{11} \mathrm{CN}_{x}(40 \mathrm{mg}), \mathrm{Ni}\left(\mathrm{NO}_{3}\right)_{2} \cdot 6 \mathrm{H}_{2} \mathrm{O}(5 \mathrm{mg})$ and $0.05 \mathrm{M} \mathrm{NaOH}$ $(10 \mathrm{~mL})$ were combined and stirred for $20 \mathrm{~h}$. The mixture was centrifuged at $7000 \mathrm{rpm}$ for $5 \mathrm{~min}$. The precipitate was then washed with water $(3 x)$ and ethanol $(3 x)$, and dried under vacuum.

[f] The $\mathrm{Ni}_{2} \mathrm{P}$ synthesis procedure was adapted to produce $\mathrm{CN}_{x} \mid \mathrm{Fe} \times \mathrm{P}_{\times} \mathrm{P}$. $\mathrm{FeCl}_{3} \cdot 6 \mathrm{H}_{2} \mathrm{O}(10 \mathrm{mg}), \mathrm{NaH}_{2} \mathrm{PO}_{2} \cdot \mathrm{H}_{2} \mathrm{O}$ $(50 \mathrm{mg})$, and $\mathrm{CN}_{\mathrm{x}}(150 \mathrm{mg})$ were mixed in a minimum of water, stirred for $1 \mathrm{~h}$ and sonicated for $1 \mathrm{~h}$. The mixture was dried under vacuum at $60^{\circ} \mathrm{C}$ and heated for $1 \mathrm{~h}$ at $200^{\circ} \mathrm{C}$ under $\operatorname{Ar}\left(\operatorname{ramp}\right.$ rate $\left.5^{\circ} \mathrm{C} \mathrm{min} \mathrm{m}^{-1}\right)$. After cooling, the powder was washed with water $(3 x)$ and ethanol $(3 \times)$, and dried under vacuum. 
Table S10. Photoreforming of a variety of substrates with $\mathrm{CN}_{x} \mid \mathrm{Ni}_{2} \mathrm{P}$. Conditions: ultra-sonicated $\mathrm{CN}_{x} \mid \mathrm{Ni}_{2} \mathrm{P} 2 \mathrm{wt} \%$ (3.2 mg), pre-treated polymer $(50 \mathrm{mg})$, aq. $\mathrm{KOH}(1 \mathrm{M}, 2 \mathrm{~mL})$, sealed photoreactor (internal volume $7.91 \mathrm{~mL}$ ) under anaerobic conditions, simulated solar light (AM 1.5G, $100 \mathrm{~mW} \mathrm{~cm} \mathrm{c}^{-2}$, $25^{\circ} \mathrm{C}$ ). Yields and activities are cumulative values. Single measurements only.

\begin{tabular}{|c|c|c|c|}
\hline Substrate & $\begin{array}{c}\text { Time } \\
\text { (h) }\end{array}$ & $\begin{array}{c}\text { Yield } \\
\left(\mu \mathrm{mol}_{2} \mathrm{gsub}^{-1}\right)\end{array}$ & $\begin{array}{c}\text { Activity } \\
\left(\mu \mathrm{mol}_{2} \mathrm{gcat}^{-1} \mathrm{~h}^{-1}\right)\end{array}$ \\
\hline \multirow{2}{*}{ PE } & 4 & 1.76 & 6.87 \\
\hline & 20 & 6.88 & 5.37 \\
\hline \multirow{2}{*}{ PET } & 4 & 3.62 & 14.1 \\
\hline & 20 & 39.9 & 31.2 \\
\hline \multirow{2}{*}{ PLA } & 4 & 4.22 & 16.5 \\
\hline & 20 & 42.1 & 32.9 \\
\hline \multirow{2}{*}{ PP } & 4 & 1.74 & 6.80 \\
\hline & 20 & 7.72 & 6.03 \\
\hline \multirow{2}{*}{ PS } & 4 & 2.32 & 9.06 \\
\hline & 20 & 6.14 & 4.80 \\
\hline \multirow{2}{*}{ PUR } & 4 & 1.22 & 4.76 \\
\hline & 20 & 7.74 & 6.05 \\
\hline \multirow{2}{*}{ Rubber } & 4 & 1.54 & 6.01 \\
\hline & 20 & 5.56 & 4.34 \\
\hline
\end{tabular}

Table S11. Photoreforming of PET and PLA with $\mathrm{CN}_{\times} \mid \mathrm{Ni}_{2} \mathrm{P}$. Conditions: ultra-sonicated $\mathrm{CN}_{\times} \mid \mathrm{Ni}_{2} \mathrm{P} 2 \mathrm{wt} \%$ (3.2 mg), pre-treated polymer ( $50 \mathrm{mg}$ ), aq. $\mathrm{KOH}(1 \mathrm{M}, 2 \mathrm{~mL}$ ), sealed photoreactor (internal volume 7.91 $\mathrm{mL}$ ) under anaerobic conditions, simulated solar light (AM 1.5G, $100 \mathrm{~mW} \mathrm{~cm}{ }^{-2}, 25^{\circ} \mathrm{C}$ ). Yields and activities are cumulative values. $\sigma$ is the standard deviation calculated from 3 samples.

\begin{tabular}{|c|c|c|c|}
\hline Description & $\begin{array}{l}\text { Time } \\
\text { (h) }\end{array}$ & $\begin{array}{c}\text { Yield } \pm \sigma \\
\left(\mu \mathrm{mol}_{2} \mathrm{~g}_{\mathrm{sub}^{-1}}\right)\end{array}$ & $\begin{array}{c}\text { Activity } \pm \sigma \\
\left(\mu \mathrm{mol}_{H_{2}} \mathrm{gcat}^{-1} \mathrm{~h}^{-1}\right)\end{array}$ \\
\hline \multirow{8}{*}{$\begin{array}{c}\text { Long-term photoreforming of } \\
\text { pre-treated PET }\end{array}$} & 2 & $3.90 \pm 0.19$ & $30.5 \pm 1.5$ \\
\hline & 4 & $6.52 \pm 0.33$ & $25.5 \pm 1.3$ \\
\hline & 20 & $33.1 \pm 1.7$ & $25.8 \pm 1.3$ \\
\hline & 25 & $42.1 \pm 3.4$ & $26.3 \pm 2.1$ \\
\hline & 27 & $45.4 \pm 2.3$ & $26.3 \pm 1.3$ \\
\hline & 44 & $72.0 \pm 6.9$ & $25.6 \pm 2.5$ \\
\hline & 46 & $73.5 \pm 7.5$ & $25.0 \pm 2.5$ \\
\hline & 50 & $82.5 \pm 7.3$ & $25.7 \pm 2.3$ \\
\hline \multirow{8}{*}{$\begin{array}{l}\text { Long-term photoreforming of } \\
\text { pre-treated PLA }\end{array}$} & 2 & $5.50 \pm 0.27$ & $43.0 \pm 2.1$ \\
\hline & 4 & $9.92 \pm 0.50$ & $38.7 \pm 1.9$ \\
\hline & 20 & $59.7 \pm 6.0$ & $46.6 \pm 4.7$ \\
\hline & 25 & $77.8 \pm 6.8$ & $48.6 \pm 4.3$ \\
\hline & 27 & $86.4 \pm 6.4$ & $50.0 \pm 3.7$ \\
\hline & 44 & $156 \pm 12$ & $55.4 \pm 4.2$ \\
\hline & 46 & $164 \pm 15$ & $55.8 \pm 5.1$ \\
\hline & 50 & $178 \pm 12$ & $55.7 \pm 3.7$ \\
\hline
\end{tabular}


Table S12. External quantum yield (EQY) measurements from photoreforming of polymers. Conditions: ultra-sonicated $\mathrm{CN}_{\times} \mid \mathrm{Ni}_{2} \mathrm{P} 2 \mathrm{wt} \%$ (3.2 mg), pre-treated polymer (50 mg), aq. $\mathrm{KOH}(1 \mathrm{M}, 2 \mathrm{~mL}$ ), all in a sealed quartz cuvette (path length $1 \mathrm{~cm}$, internal volume $3.83 \mathrm{~mL}$ ) under anaerobic conditions. Samples were irradiated with monochromatic light $(\lambda=430 \mathrm{~nm}$, full-width at half maximum: 5 , intensity taken as the average of the intensities measured at the beginning and end of the experiments) over an area of $0.28 \mathrm{~cm}^{2} . \sigma$ is the standard deviation calculated from the 3 listed samples.

\begin{tabular}{|c|c|c|c|c|c|c|}
\hline Substrate & $\begin{array}{l}\text { Substrate } \\
\text { Conditions }\end{array}$ & Time (h) & $\begin{array}{l}\text { Light Intensity } \\
\quad\left(\mathrm{mW} \mathrm{cm}^{-2}\right)\end{array}$ & $\begin{array}{c}\mathrm{H}_{2} \\
(\mu \mathrm{mol})\end{array}$ & $\begin{array}{c}\text { EQY } \\
(\%)\end{array}$ & $\begin{array}{c}\text { Average } \pm \sigma \\
\text { EQY (\%) }\end{array}$ \\
\hline PLA & $\begin{array}{c}25 \mathrm{mg} \mathrm{mL}^{-1} \text {, } \\
\text { pre-treated }\end{array}$ & 24 & $\begin{array}{l}0.64 \pm 0.10 \\
0.95 \pm 0.07 \\
0.90 \pm 0.01\end{array}$ & $\begin{array}{l}0.022 \\
0.043 \\
0.052\end{array}$ & $\begin{array}{l}0.078 \\
0.097 \\
0.128\end{array}$ & $0.101 \pm 0.018$ \\
\hline PET & $\begin{array}{r}25 \mathrm{mg} \mathrm{mL}^{-1} \text {, } \\
\text { pre-treated }\end{array}$ & 24 & $\begin{array}{c}0.50 \pm 0.08 \\
0.75 \pm 011 \\
0.90 \pm 0.01\end{array}$ & $\begin{array}{l}0.009 \\
0.009 \\
0.014\end{array}$ & $\begin{array}{l}0.041 \\
0.028 \\
0.036\end{array}$ & $0.035 \pm 0.005$ \\
\hline
\end{tabular}

Table S13. Stoichiometric $\mathrm{H}_{2}$ conversion calculations. Conditions: ultra-sonicated $\mathrm{CN}_{*} \mid \mathrm{Ni}_{2} \mathrm{P} 2 \mathrm{wt} \%$ (3.2 $\mathrm{mg}$ ), polymer $(5 \mathrm{mg})$, aq. $\mathrm{KOH}(1 \mathrm{M}$ or $10 \mathrm{M}, 2 \mathrm{~mL}$ ), sealed photoreactor (internal volume $7.91 \mathrm{~mL}$ ) under anaerobic conditions, simulated solar light (AM 1.5G, $100 \mathrm{~mW} \mathrm{~cm}{ }^{-2}, 25^{\circ} \mathrm{C}$ ). Yields and activities are cumulative values. $\sigma$ is the standard deviation calculated from 3 samples.

\begin{tabular}{|c|c|c|c|c|c|}
\hline Description & Substrate & $\begin{array}{c}N_{100 \%} \\
\left(\mathrm{~mol}_{\mathrm{H}_{2}} \mathrm{~mol}_{\mathrm{sub}^{-1}}{ }^{-1}\right)\end{array}$ & $\begin{array}{c}\text { Time } \\
\text { (h) }\end{array}$ & $\begin{array}{c}N_{\text {yield }} \pm \sigma \\
\left(\text { mol }_{H_{2}} \text { molsub }^{-1}\right)\end{array}$ & $\begin{array}{c}\text { Conversion } \pm \sigma \\
(\%)\end{array}$ \\
\hline \multirow{8}{*}{$\begin{array}{c}\mathrm{H}_{2} \text { Conversion in } \\
1 \mathrm{M} \mathrm{KOH}\end{array}$} & \multirow{4}{*}{$\begin{array}{c}\text { PET, } \\
26.0 \mu \mathrm{mol}\end{array}$} & \multirow{4}{*}{$5.0^{[a]}$} & 72 & $0.027 \pm 0.003$ & $0.54 \pm 0.06$ \\
\hline & & & 96 & $0.040 \pm 0.004$ & $0.80 \pm 0.08$ \\
\hline & & & 144 & $0.121 \pm 0.015$ & $2.42 \pm 0.30$ \\
\hline & & & 192 & $0.219 \pm 0.029$ & $4.38 \pm 0.58$ \\
\hline & \multirow{4}{*}{$\begin{array}{c}\text { PLA, } \\
69.4 \mu \mathrm{mol}\end{array}$} & \multirow{4}{*}{6.0} & 72 & $0.016 \pm 0.002$ & $0.27 \pm 0.03$ \\
\hline & & & 96 & $0.026 \pm 0.004$ & $0.43 \pm 0.07$ \\
\hline & & & 144 & $0.061 \pm 0.012$ & $1.02 \pm 0.20$ \\
\hline & & & 192 & $0.097 \pm 0.015$ & $1.62 \pm 0.25$ \\
\hline \multirow{8}{*}{$\begin{array}{c}\mathrm{H}_{2} \text { Conversion in } \\
10 \mathrm{M} \mathrm{KOH}\end{array}$} & \multirow{4}{*}{$\begin{array}{c}\text { PET, } \\
26.0 \mu \mathrm{mol}\end{array}$} & \multirow{4}{*}{$5.0^{[\mathrm{a}]}$} & 72 & $0.284 \pm 0.014$ & $5.68 \pm 0.28$ \\
\hline & & & 96 & $0.385 \pm 0.037$ & $7.70 \pm 0.74$ \\
\hline & & & 144 & $0.932 \pm 0.114$ & $18.6 \pm 2.3$ \\
\hline & & & 192 & $1.23 \pm 0.16$ & $24.5 \pm 3.3$ \\
\hline & \multirow{4}{*}{$\begin{array}{c}\text { PLA, } \\
69.4 \mu \mathrm{mol}\end{array}$} & \multirow{4}{*}{6.0} & 72 & $0.079 \pm 0.005$ & $1.32 \pm 0.08$ \\
\hline & & & 96 & $0.135 \pm 0.012$ & $2.25 \pm 0.20$ \\
\hline & & & 144 & $0.295 \pm 0.033$ & $4.92 \pm 0.55$ \\
\hline & & & 192 & $0.401 \pm 0.048$ & $6.68 \pm 0.80$ \\
\hline
\end{tabular}

[a] This number assumes that only the ethylene glycol component of PET is oxidized. 
Table S14. Photoreforming with other photocatalysts. $\mathrm{CN}_{x} \mid \mathrm{Ni}_{2} \mathrm{P}$ is included for ease of comparison. Conditions: catalyst $(3.2 \mathrm{mg})$, pre-treated polymer $(50 \mathrm{mg})$, aq. $\mathrm{KOH}(1 \mathrm{M}, 2 \mathrm{~mL})$, sealed photoreactor (internal volume $7.91 \mathrm{~mL}$ ) under anaerobic conditions, simulated solar light (AM 1.5G, $100 \mathrm{~mW} \mathrm{~cm}^{-2}$, $25^{\circ} \mathrm{C}$ ). Yields and activities are cumulative values. $\sigma$ is the standard deviation calculated from 3 samples.

\begin{tabular}{|c|c|c|c|c|c|}
\hline Description & Catalyst & Substrate & $\begin{array}{l}\text { Time } \\
\text { (h) }\end{array}$ & $\begin{array}{c}\text { Yield } \pm \sigma \\
\left(\mu \mathrm{mol}_{2} \mathrm{gsub}^{-1}\right)\end{array}$ & $\begin{array}{c}\text { Activity } \pm \sigma \\
\left(\mu \mathrm{mol}_{2} \mathrm{gcat}^{-1} \mathrm{~h}^{-1}\right)\end{array}$ \\
\hline & \multirow{8}{*}{$\begin{array}{c}\mathrm{CN}_{\times} \mid \mathrm{Ni}_{2} \mathrm{P} \\
2 \mathrm{wt} \%\end{array}$} & \multirow{4}{*}{ PET } & 4 & $6.52 \pm 0.33$ & $25.5 \pm 1.3$ \\
\hline & & & 20 & $33.1 \pm 1.7$ & $25.8 \pm 1.3$ \\
\hline & & & 25 & $42.1 \pm 3.4$ & $26.3 \pm 2.1$ \\
\hline & & & 44 & $72.0 \pm 6.9$ & $25.6 \pm 2.5$ \\
\hline & & \multirow{4}{*}{ PLA } & 4 & $9.92 \pm 0.50$ & $38.7 \pm 1.9$ \\
\hline & & & 20 & $59.7 \pm 6.0$ & $46.6 \pm 4.7$ \\
\hline & & & 25 & $77.8 \pm 6.8$ & $48.6 \pm 4.3$ \\
\hline & & & 44 & $156 \pm 12$ & $55.4 \pm 4.2$ \\
\hline \multirow{20}{*}{$\begin{array}{c}\text { Alternative } \\
\text { photocatalysts }\end{array}$} & \multirow{2}{*}{$\begin{array}{c}2 \mathrm{wt} \% \mathrm{Ni}_{2} \mathrm{P} \\
\text { powder }+\mathrm{CN}_{x}\end{array}$} & \multirow{2}{*}{ PET } & 4 & $0.24 \pm 0.013$ & $0.937 \pm 0.051$ \\
\hline & & & 20 & $10.5 \pm 0.7$ & $8.22 \pm 0.55$ \\
\hline & \multirow{8}{*}{$\begin{array}{l}{ }_{2}^{\mathrm{H}_{2} \mathrm{~N}} \mathrm{CN}_{\times} \mid \mathrm{Ni}_{2} \mathrm{P} \\
2 \mathrm{wt} \%\end{array}$} & \multirow{4}{*}{ PET } & 4 & $10.5 \pm 2.1$ & $41.1 \pm 8.2$ \\
\hline & & & 20 & $34.3 \pm 3.1$ & $26.8 \pm 2.4$ \\
\hline & & & 25 & $39.5 \pm 3.2$ & $24.7 \pm 2.0$ \\
\hline & & & 44 & $42.5 \pm 5.5$ & $15.1 \pm 2.0$ \\
\hline & & \multirow{4}{*}{ PLA } & 4 & $21.1 \pm 2.8$ & $82.5 \pm 10.9$ \\
\hline & & & 20 & $81.2 \pm 6.1$ & $63.4 \pm 4.7$ \\
\hline & & & 25 & $98.6 \pm 7.8$ & $61.6 \pm 4.8$ \\
\hline & & & 44 & $141 \pm 18$ & $50.2 \pm 6.4$ \\
\hline & \multirow{4}{*}{$\begin{array}{c}\mathrm{TiO}_{2} \mid \mathrm{Ni}_{2} \mathrm{P} \\
2 \mathrm{wt} \%\end{array}$} & \multirow{2}{*}{ PET } & 4 & $0.900 \pm 0.105$ & $3.52 \pm 0.41$ \\
\hline & & & 20 & $13.8 \pm 2.4$ & $10.8 \pm 1.9$ \\
\hline & & \multirow{2}{*}{ PLA } & 4 & $8.48 \pm 5.12$ & $33.1 \pm 20.0$ \\
\hline & & & 20 & $54.1 \pm 9.4$ & $42.3 \pm 7.4$ \\
\hline & \multirow{4}{*}{$\mathrm{CN}_{x} \mid \mathrm{Pt}, 2 \mathrm{wt} \%$} & \multirow{2}{*}{ PET } & 4 & $29.7 \pm 6.1$ & $116 \pm 24$ \\
\hline & & & 20 & $96.2 \pm 4.8$ & $75.2 \pm 3.7$ \\
\hline & & \multirow{2}{*}{ PLA } & 4 & $20.0 \pm 1.3$ & $156 \pm 10$ \\
\hline & & & 20 & $180 \pm 17$ & $281 \pm 26$ \\
\hline & \multirow{2}{*}{$\begin{array}{c}\mathrm{CN}_{x}-\mathrm{P} \mid \mathrm{Pt} \\
2 \mathrm{wt} \%\end{array}$} & \multirow{2}{*}{ PET } & 4 & $16.5 \pm 0.8$ & $64.5 \pm 3.2$ \\
\hline & & & 20 & $92.6 \pm 11.5$ & $72.4 \pm 9.0$ \\
\hline \multirow{8}{*}{$\begin{array}{c}\text { Alternative } \\
\text { photocatalyst } \\
\text { irradiated with } \lambda \\
>420 \mathrm{~nm} \text { filter }\end{array}$} & \multirow{4}{*}{$\begin{array}{c}\mathrm{TiO}_{2} \mid \mathrm{Ni}_{2} \mathrm{P}, \\
2 \mathrm{wt} \%\end{array}$} & \multirow{2}{*}{ PET } & 4 & $0.0 \pm 0.0$ & $0.0 \pm 0.0$ \\
\hline & & & 20 & $0.0 \pm 0.0$ & $0.0 \pm 0.0$ \\
\hline & & \multirow{2}{*}{ PLA } & 4 & $0.0 \pm 0.0$ & $0.0 \pm 0.0$ \\
\hline & & & 20 & $0.0 \pm 0.0$ & $0.0 \pm 0.0$ \\
\hline & \multirow{4}{*}{$\begin{array}{c}\mathrm{H}_{2} \mathrm{~N}_{\mathrm{CN}} \mathrm{N}_{\times} \mid \mathrm{Ni}_{2} \mathrm{P} \\
2 \mathrm{wt} \%\end{array}$} & \multirow{2}{*}{ PET } & 4 & $0.0 \pm 0.0$ & $0.0 \pm 0.0$ \\
\hline & & & 20 & $0.720 \pm 0.480$ & $0.562 \pm 0.374$ \\
\hline & & \multirow{2}{*}{ PLA } & 4 & $0.320 \pm 0.016$ & $0.205 \pm 0.010$ \\
\hline & & & 20 & $7.20 \pm 0.36$ & $3.25 \pm 0.30$ \\
\hline
\end{tabular}


Table S15. Comparison of the current work to other reported catalysts for polymer photoreforming. Samples referenced as [12] were irradiated with a $500 \mathrm{~W}$ Xe lamp. Samples referenced as * or [13] were irradiated with a $1000 \mathrm{~W}$ Xe lamp (AM 1.5G, $100 \mathrm{~mW} \mathrm{~cm}^{-2}, 25^{\circ} \mathrm{C}$ ).

\begin{tabular}{|c|c|c|c|c|c|c|}
\hline Catalyst & Substrate $\mathrm{e}^{[\mathrm{a}]}$ & [base] $^{[\mathrm{b}]}$ & $\begin{array}{c}\text { Time } \\
\text { (h) }\end{array}$ & $\begin{array}{c}\text { Yield } \pm \sigma \\
\left(\mu \mathrm{mol}_{\mathrm{H}_{2}} \mathrm{~g}_{\mathrm{sub}^{-1}}\right)\end{array}$ & $\begin{array}{c}\text { Activity } \pm \sigma \\
\left(\mu \mathrm{mol}_{\mathrm{H}_{2}} \mathrm{~g}_{\left.\mathrm{cat}^{-1} \mathrm{~h}^{-1}\right)}\right.\end{array}$ & Ref \\
\hline $\mathrm{TiO}_{2} \mid \mathrm{Pt}, 5 \%[\mathrm{c}, \mathrm{d}]$ & PE & $5 \mathrm{M}$ & 10 & 620 & 31.0 & 12 \\
\hline $\mathrm{TiO}_{2} \mid \mathrm{Pt}, 5 \%$ & PVC & $5 \mathrm{M}$ & 10 & 300 & 28.7 & 12 \\
\hline $\mathrm{TiO}_{2} \mid \mathrm{Pt}, 5 \%$ & PET & $10 \mathrm{M}$ & 20 & $1220 \pm 110$ & $153 \pm 14$ & 13 \\
\hline $\mathrm{CdS} / \mathrm{CdO}_{x}[\mathrm{e}]$ & PET & $10 \mathrm{M}$ & 20 & $460 \pm 58$ & $4810 \pm 600$ & 13 \\
\hline $\mathrm{H}_{2} \mathrm{~N}_{\mathrm{CN}} \mathrm{N}_{\mathrm{x}} \mid \mathrm{Ni}_{2} \mathrm{P}, 2 \%{ }^{[\mathrm{f}]}$ & PET & $10 \mathrm{M}$ & 20 & $141 \pm 16$ & $110 \pm 12$ & * \\
\hline $\mathrm{TiO}_{2} \mid \mathrm{Ni}_{2} \mathrm{P}, 2 \%$ & PET & $10 \mathrm{M}$ & 20 & $119 \pm 8$ & $93.0 \pm 6.6$ & * \\
\hline $\mathrm{CN}_{x} \mid \mathrm{Ni}_{2} \mathrm{P}, 2 \%$ & PET & $10 \mathrm{M}$ & 20 & $111 \pm 8$ & $83.2 \pm 6.0$ & * \\
\hline $\mathrm{CN}_{\mathrm{x}} \mid \mathrm{Pt}, 2 \%$ & PET & $10 \mathrm{M}$ & 20 & $104 \pm 4$ & $81.1 \pm 3.4$ & * \\
\hline $\mathrm{CdS} / \mathrm{CdO}_{\mathrm{x}}$ & PET & $1 \mathrm{M}$ & 20 & $132 \pm 6$ & $2210 \pm 110$ & 13 \\
\hline $\mathrm{CN}_{\mathrm{x}} \mid \mathrm{Pt}, 2 \%$ & PET & $1 \mathrm{M}$ & 20 & $96.2 \pm 4.8$ & $75.2 \pm 3.7$ & * \\
\hline${ }^{\mathrm{H}_{2} \mathrm{~N}} \mathrm{CN}_{\mathrm{x}} \mid \mathrm{Ni}_{2} \mathrm{P}, 2 \%$ & PET & $1 \mathrm{M}$ & 20 & $34.3 \pm 3.1$ & $26.8 \pm 2.4$ & * \\
\hline $\mathrm{CN}_{\times} \mid \mathrm{Ni}_{2} \mathrm{P}, 2 \%$ & PET & $1 \mathrm{M}$ & 20 & $33.1 \pm 1.7$ & $25.8 \pm 1.3$ & * \\
\hline $\mathrm{TiO}_{2} \mid \mathrm{Ni}_{2} \mathrm{P}, 2 \%^{[\mathrm{f}]}$ & PET & $1 \mathrm{M}$ & 20 & $13.8 \pm 2.4$ & $10.8 \pm 1.9$ & * \\
\hline $\mathrm{CdS} / \mathrm{CdO}$ & PLA & $10 \mathrm{M}$ & 20 & $2590 \pm 690$ & $63000 \pm 16800$ & 13 \\
\hline${ }^{\mathrm{H}_{2} \mathrm{~N}} \mathrm{CN}_{\mathrm{x}} \mid \mathrm{Ni}_{2} \mathrm{P}, 2 \%$ & PLA & $10 \mathrm{M}$ & 20 & $427 \pm 21$ & $333 \pm 17$ & * \\
\hline $\mathrm{TiO}_{2} \mid \mathrm{Pt}, 5 \%$ & PLA & $10 \mathrm{M}$ & 20 & $358 \pm 53$ & $89 \pm 13$ & 13 \\
\hline $\mathrm{CN}_{\mathrm{x}} \mid \mathrm{Pt}, 2 \%$ & PLA & $10 \mathrm{M}$ & 20 & $314 \pm 16$ & $491 \pm 24$ & * \\
\hline $\mathrm{TiO}_{2} \mid \mathrm{Ni}_{2} \mathrm{P}, 2 \%$ & PLA & $10 \mathrm{M}$ & 20 & $220 \pm 28$ & $173 \pm 22$ & * \\
\hline $\mathrm{CN}_{\mathrm{x}} \mid \mathrm{Ni}_{2} \mathrm{P}, 2 \%$ & PLA & $10 \mathrm{M}$ & 20 & $211 \pm 10$ & $165 \pm 8$ & * \\
\hline $\mathrm{CN}_{\mathrm{x}} \mid \mathrm{Pt}, 2 \%$ & PLA & $1 \mathrm{M}$ & 20 & $180 \pm 17$ & $281 \pm 26$ & * \\
\hline${ }^{\mathrm{H}_{2} \mathrm{~N}} \mathrm{CN}_{\mathrm{x}} \mid \mathrm{Ni}_{2} \mathrm{P}, 2 \%$ & PLA & $1 \mathrm{M}$ & 20 & $81.2 \pm 6.1$ & $63.4 \pm 4.8$ & * \\
\hline $\mathrm{CN}_{\times} \mid \mathrm{Ni}_{2} \mathrm{P}, 2 \%$ & PLA & $1 \mathrm{M}$ & 20 & $59.7 \pm 6.0$ & $46.6 \pm 4.7$ & * \\
\hline $\mathrm{CdS} / \mathrm{CdO}_{\mathrm{x}}$ & PLA & $1 \mathrm{M}$ & 20 & $56.6 \pm 8.9$ & $839 \pm 132$ & 13 \\
\hline $\mathrm{TiO}_{2} \mid \mathrm{Ni}_{2} \mathrm{P}, 2 \%$ & PLA & $1 \mathrm{M}$ & 20 & $54.1 \pm 9.4$ & $42.3 \pm 7.4$ & * \\
\hline
\end{tabular}

[a] All polymer substrates except for PE and PVC were pre-treated prior to use (25 $\left.\mathrm{mg} \mathrm{mL}^{-1}\right)$.

[b] PE and PVC were run in $30 \mathrm{~mL}$ of base, whereas all other samples were run in $2 \mathrm{~mL}$ of base.

[c] Percentages indicate wt\% of the co-catalyst.

[d] $300 \mathrm{mg} \mathrm{TiO} 2 \mid \mathrm{Pt}$ used per sample.

[e] $1 \mathrm{nmol} \mathrm{CdS} / \mathrm{CdO}_{x}$ used per sample.

[f] $3.2 \mathrm{mg}^{\mathrm{H} 2 \mathrm{~N}} \mathrm{CN}_{\mathrm{x}}\left|\mathrm{Ni}_{2} \mathrm{P}, \mathrm{CN}_{\mathrm{x}}\right| \mathrm{Ni}_{2} \mathrm{P}, \mathrm{CN}_{\mathbf{x}} \mid \mathrm{Pt}$ or $\mathrm{TiO}_{2} \mid \mathrm{Ni}_{2} \mathrm{P}$ used per sample.

* This work. 
Table S16. Photoreforming of oxidation intermediates with $\mathrm{CN}_{x} \mid \mathrm{Ni}_{2} \mathrm{P}$. Conditions: $\mathrm{CN}_{\times} \mid \mathrm{Ni}_{2} \mathrm{P} 2 \mathrm{wt} \%$ (3.2 $\mathrm{mg}$ ), substrate $(50 \mathrm{mg})$, aq. $\mathrm{KOH}(1 \mathrm{M}, 2 \mathrm{~mL})$, sealed photoreactor (internal volume $7.91 \mathrm{~mL}$ ) under anaerobic conditions, simulated solar light (AM 1.5G, $100 \mathrm{~mW} \mathrm{~cm}-2,25^{\circ} \mathrm{C}$ ). Yields and activities are cumulative values. $\sigma$ is the standard deviation calculated from 3 samples, unless stated otherwise.

\begin{tabular}{|c|c|c|c|}
\hline Substrate & $\begin{array}{l}\text { Time } \\
\text { (h) }\end{array}$ & $\begin{array}{c}\text { Yield } \pm \sigma \\
\left(\mu \mathrm{mol}_{\mathrm{H}_{2}} \mathrm{~g}_{\mathrm{sub}^{-1}}\right)\end{array}$ & $\begin{array}{c}\text { Activity } \pm \sigma \\
\left(\mu \mathrm{mol}_{\mathrm{H}_{2}} \mathrm{gcat}^{-1} \mathrm{~h}^{-1}\right)\end{array}$ \\
\hline \multirow{2}{*}{ Acetate $^{[a]}$} & 4 & $1.12 \pm 0.14$ & $4.37 \pm 0.56$ \\
\hline & 20 & $3.22 \pm 0.40$ & $2.51 \pm 0.31$ \\
\hline \multirow{2}{*}{ Ethylene glycol } & 4 & $12.9 \pm 0.6$ & $50.5 \pm 2.5$ \\
\hline & 20 & $58.9 \pm 7.1$ & $46.0 \pm 5.6$ \\
\hline \multirow{2}{*}{ Formate ${ }^{[a]}$} & 4 & $4.30 \pm 1.80$ & $16.8 \pm 7.0$ \\
\hline & 20 & $17.7 \pm 1.3$ & $13.8 \pm 1.0$ \\
\hline \multirow{2}{*}{ Glycolate ${ }^{[a]}$} & 4 & $3.58 \pm 0.85$ & $14.0 \pm 3.32$ \\
\hline & 20 & $14.6 \pm 2.1$ & $11.4 \pm 1.7$ \\
\hline \multirow{2}{*}{ Glyoxal[a] } & 4 & $10.6 \pm 0.5$ & $41.3 \pm 2.1$ \\
\hline & 20 & $50.2 \pm 6.2$ & $39.2 \pm 4.9$ \\
\hline \multirow{2}{*}{ Lactate } & 4 & $6.20 \pm 0.77$ & $24.2 \pm 3.0$ \\
\hline & 20 & $40.4 \pm 3.4$ & $31.6 \pm 2.7$ \\
\hline \multirow{2}{*}{ Terephthalate } & 4 & $0.0 \pm 0.0$ & $0.0 \pm 0.0$ \\
\hline & 20 & $0.0 \pm 0.0$ & $0.0 \pm 0.0$ \\
\hline
\end{tabular}

${ }^{[a]} \sigma$ obtained from two samples.

Table S17. Re-use of $\mathrm{CN}_{*} \mid \mathrm{Ni}_{2} \mathrm{P}$ for photoreforming of PET. Conditions: previously used $\mathrm{CN}_{*} \mid \mathrm{Ni}_{2} \mathrm{P} 2 \mathrm{wt} \%$ after centrifugation, washing and drying (3.2 mg), pre-treated PET (50 mg), aq. $\mathrm{KOH}(1 \mathrm{M}, 2 \mathrm{~mL}$ ), sealed photoreactor (internal volume $7.91 \mathrm{~mL}$ ) under anaerobic conditions, simulated solar light (AM 1.5G, 100 $\mathrm{mW} \mathrm{cm}{ }^{-2}, 25^{\circ} \mathrm{C}$ ). Yields and activities are cumulative values. $\sigma$ is the standard deviation calculated from 3 samples.

\begin{tabular}{ccc}
\hline $\begin{array}{c}\text { Time } \\
(\mathbf{h})\end{array}$ & $\begin{array}{c}\text { Yield } \pm \sigma \\
\left(\mu \mathrm{mol}_{2} \mathrm{gsub}^{-1}\right)\end{array}$ & $\begin{array}{c}\text { Activity } \pm \sigma \\
\left(\mu \mathrm{mol}_{\mathrm{H}_{2}} \mathbf{g}_{\mathrm{cat}^{-1}} \mathbf{h}^{-1}\right)\end{array}$ \\
\hline 4 & $0.60 \pm 0.03$ & $2.34 \pm 0.12$ \\
20 & $14.6 \pm 0.7$ & $11.4 \pm 0.6$ \\
\hline
\end{tabular}

Table S18. Quantification of the organic oxidation products formed from glyoxal and formate after 24 $\mathrm{h}$ of photoreforming. Maleic acid in $\mathrm{D}_{2} \mathrm{O}$ was used as an internal standard.

\begin{tabular}{lcc}
\hline & Organic compound & Quantity (nmol) \\
\hline & Acetate & 260 \\
Photoreforming of glyoxal & Formate & 1640 \\
& Glycolate & 6550 \\
\hline Photoreforming of formate & Acetate & 115 \\
\hline
\end{tabular}


Table S19. Photoreforming of different quantities of polyester microfibers. Conditions: $\mathrm{CN}_{\mathbf{x}} \mid \mathrm{Ni}_{2} \mathrm{P} 2 \mathrm{wt} \%$ (3.2 $\mathrm{mg}$ ), pre-treated fibers, aqueous $\mathrm{KOH}(1 \mathrm{M}, 2 \mathrm{~mL}$ ), sealed photoreactor (internal volume $7.91 \mathrm{~mL}$ ) under anaerobic conditions, simulated solar light (AM 1.5G, $100 \mathrm{~mW} \mathrm{~cm}^{-2}, 25^{\circ} \mathrm{C}$ ). Yields and activities are cumulative values. Single measurements only.

\begin{tabular}{|c|c|c|c|}
\hline $\begin{array}{l}\text { Substrate Concentration } \\
\left(\mathrm{mg} \mathrm{mL}^{-1}\right)\end{array}$ & $\begin{array}{c}\text { Time } \\
\text { (h) }\end{array}$ & $\begin{array}{c}\text { Yield } \\
\left(\mu \mathrm{mol}_{H_{2}} \mathrm{~g}_{\text {sub }^{-1}}\right)\end{array}$ & $\begin{array}{c}\text { Activity } \\
\left(\mu \mathrm{mol}_{H_{2}} \mathrm{gcat}^{-1} \mathrm{~h}^{-1}\right)\end{array}$ \\
\hline \multirow{2}{*}{2.5} & 4 & 0.0 & 0.0 \\
\hline & 20 & 0.0 & 0.0 \\
\hline \multirow{2}{*}{0.5} & 4 & 0.0 & 0.0 \\
\hline & 20 & 0.0 & 0.0 \\
\hline \multirow{2}{*}{0.25} & 4 & 0.0 & 0.0 \\
\hline & 20 & 0.0 & 0.0 \\
\hline
\end{tabular}

Table S20. Long-term photoreforming of real-world waste at small and large scales. Conditions: $\mathrm{CN}_{\mathrm{x}} \mid \mathrm{Ni}_{2} \mathrm{P} 2 \mathrm{wt} \%$ (3.2 $\mathrm{mg}$ for small scale, or $170 \mathrm{mg}$ for up-scaled), pre-treated polymer $\left(5 \mathrm{mg} \mathrm{mL}^{-1}\right.$ microfibers, $25 \mathrm{mg} \mathrm{mL}^{-1}$ bottle, $5 \mathrm{mg} \mathrm{mL}^{-1}$ oil), aq. $\mathrm{KOH}(1 \mathrm{M}, 2 \mathrm{~mL}$ for small scale, or $120 \mathrm{~mL}$ for upscaled), sealed photoreactor (internal volume $7.91 \mathrm{~mL}$ for small scale, $190 \mathrm{~mL}$ for up-scaled) under anaerobic conditions, simulated solar light (AM 1.5G, $100 \mathrm{~mW} \mathrm{~cm}{ }^{-2}, 25^{\circ} \mathrm{C}$ ). Yields and activities are cumulative values. $\sigma$ is the standard deviation calculated from 3 samples.

\begin{tabular}{|c|c|c|c|}
\hline Description & $\begin{array}{c}\text { Time } \\
\text { (h) }\end{array}$ & $\begin{array}{c}\text { Yield } \pm \sigma \\
\left(\mu \mathrm{mol}_{2} \mathrm{~g}_{\text {sub }^{-1}}\right)\end{array}$ & $\begin{array}{c}\text { Activity } \pm \sigma \\
\left(\mu \mathrm{mol}_{2} \mathrm{gcat}^{-1} \mathrm{~h}^{-1}\right)\end{array}$ \\
\hline \multirow{5}{*}{$\begin{array}{l}\text { Long-term photoreforming of pre- } \\
\text { treated microfibers }\end{array}$} & 24 & $17.6 \pm 2.3$ & $2.29 \pm 0.30$ \\
\hline & 48 & $31.2 \pm 3.5$ & $2.03 \pm 0.23$ \\
\hline & 72 & $43.4 \pm 2.9$ & $1.88 \pm 0.12$ \\
\hline & 96 & $63.3 \pm 4.4$ & $2.06 \pm 0.14$ \\
\hline & 120 & $104 \pm 10$ & $2.67 \pm 0.25$ \\
\hline \multirow{5}{*}{$\begin{array}{l}\text { Long-term photoreforming of pre- } \\
\text { treated bottle }\end{array}$} & 24 & $4.38 \pm 0.54$ & $2.85 \pm 0.35$ \\
\hline & 48 & $8.64 \pm 0.43$ & $2.81 \pm 0.14$ \\
\hline & 72 & $11.6 \pm 0.5$ & $2.52 \pm 0.12$ \\
\hline & 96 & $16.1 \pm 1.0$ & $2.61 \pm 0.17$ \\
\hline & 120 & $22.0 \pm 1.3$ & $2.87 \pm 0.16$ \\
\hline \multirow{5}{*}{$\begin{array}{l}\text { Long-term photoreforming of pre- } \\
\text { treated bottle }+ \text { oil }\end{array}$} & 24 & $2.40 \pm 0.16$ & $1.87 \pm 0.12$ \\
\hline & 48 & $5.23 \pm 1.08$ & $2.04 \pm 0.42$ \\
\hline & 72 & $6.47 \pm 0.32$ & $1.68 \pm 0.08$ \\
\hline & 96 & $8.27 \pm 0.55$ & $1.61 \pm 0.11$ \\
\hline & 120 & $11.4 \pm 1.2$ & $1.78 \pm 0.19$ \\
\hline \multirow{5}{*}{$\begin{array}{l}\text { Up-scaled photoreforming of pre- } \\
\text { treated microfibers }{ }^{[a]}\end{array}$} & 24 & 18.3 & 2.69 \\
\hline & 48 & 28.5 & 2.09 \\
\hline & 72 & 37.7 & 1.85 \\
\hline & 96 & 46.1 & 1.69 \\
\hline & 120 & 53.5 & 1.57 \\
\hline
\end{tabular}

[a] Values from a single experiment. 


\section{Supplementary Figures}

(a)

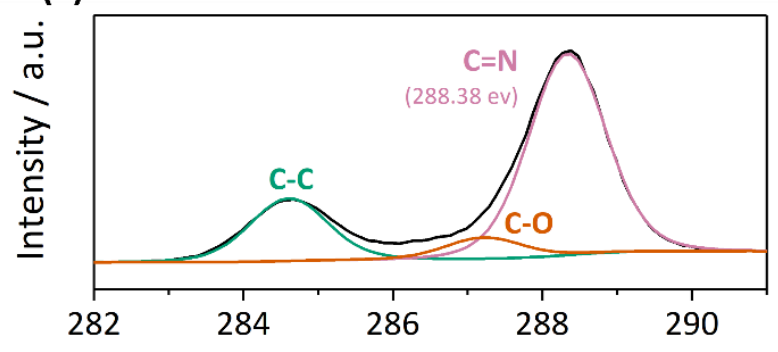

(c) (b)

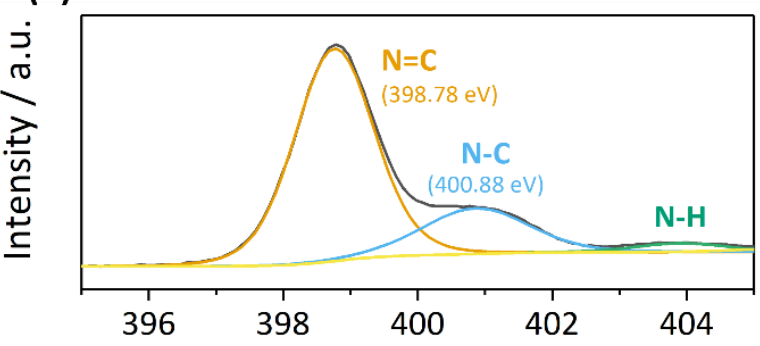

Binding Energy / eV

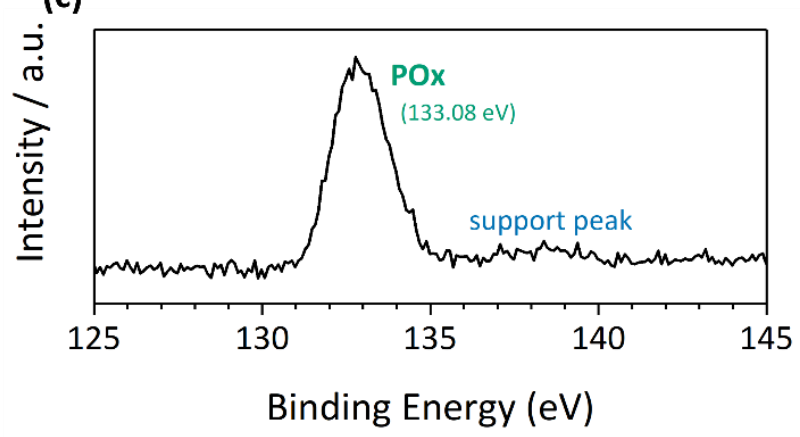

Figure S1. X-ray photoelectron spectroscopy (XPS) spectra of the (a) $\mathrm{C}_{1 \mathrm{~s}}$, (b) $\mathrm{N}_{1 \mathrm{~s}}$, and (c) $\mathrm{P}_{2 p}$ edges of $\mathrm{CN}_{x}-\mathrm{P}$. $\mathrm{CN}_{\mathrm{x}}-\mathrm{P}$ was produced according to the same synthesis procedure utilized for $\mathrm{CN}_{\mathrm{x}} \mid \mathrm{Ni}_{2} \mathrm{P}$, but without the addition of the Ni precursor. These data (along with ICP results in Table S1), suggest that excess $\mathrm{P}$ content in the $\mathrm{CN}_{\mathrm{x}} \mid \mathrm{Ni}_{2} \mathrm{P}$ photocatalyst can be attributed to residual $\mathrm{PO}_{x}$ from the co-catalyst synthesis that adheres to the $\mathrm{CN}_{\mathrm{x}}$ surface.
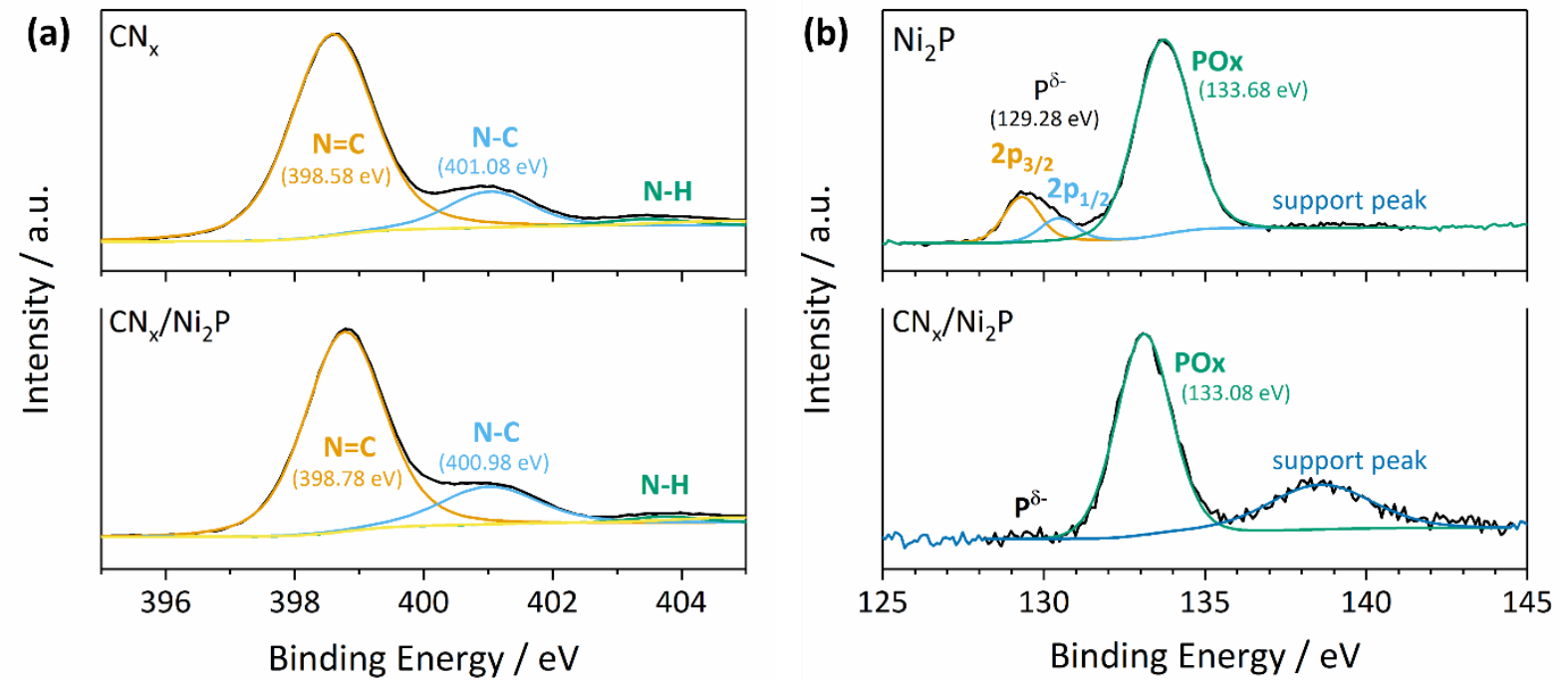

Figure S2. X-ray photoelectron spectroscopy (XPS) spectra of the (a) $\mathrm{N}_{1 s}$ edge of $\mathrm{CN}_{\mathrm{x}}$ and $\mathrm{CN}_{\mathrm{x}} \mid \mathrm{Ni}_{2} \mathrm{P}(2$ wt $\%$ ), and (b) $\mathrm{P}_{2 p}$ edge of $\mathrm{Ni}_{2} \mathrm{P}$ and $\mathrm{CN}_{x} \mid \mathrm{Ni}_{2} \mathrm{P}$ (2 wt\%). 

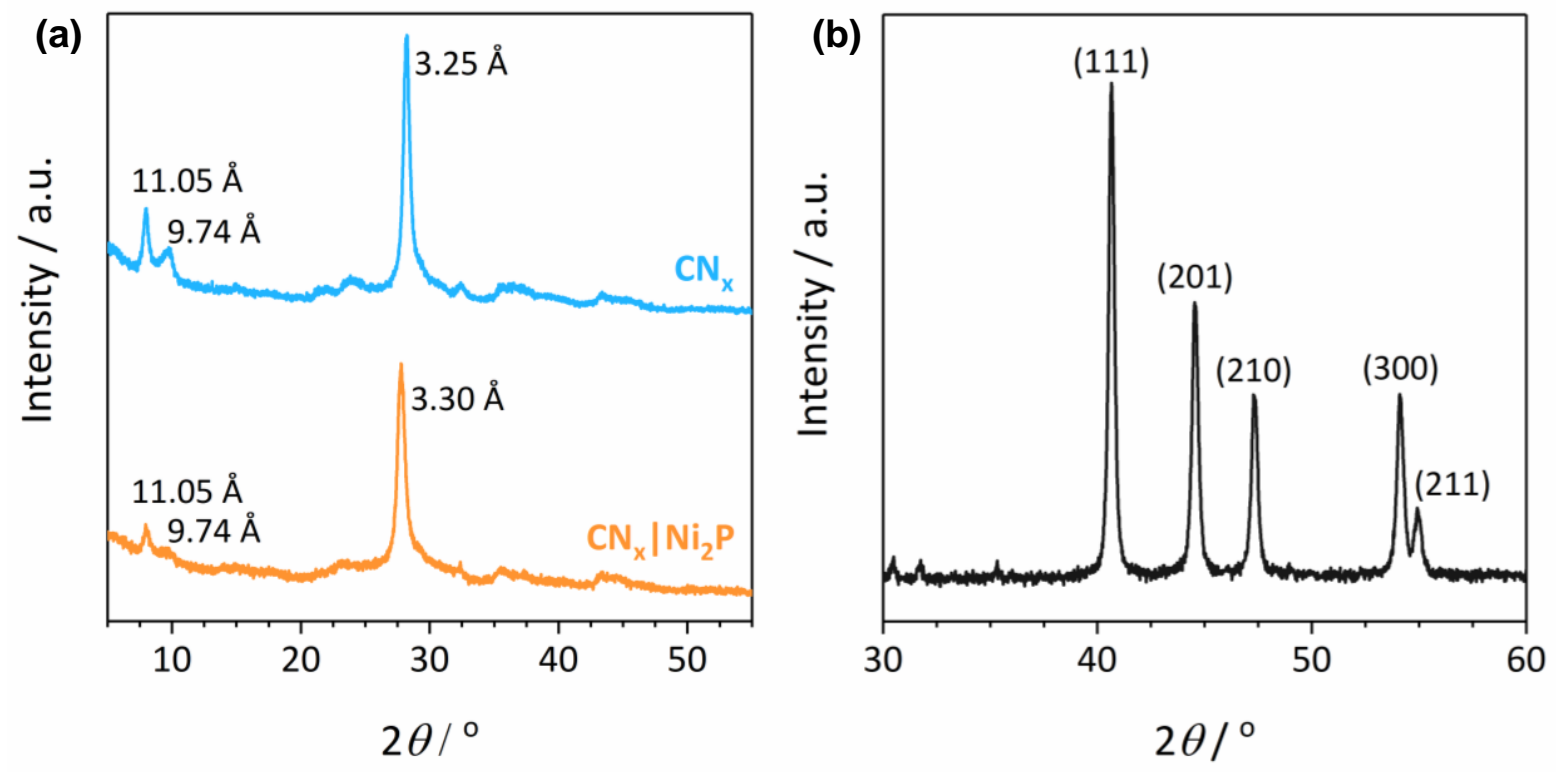

Figure S3. Powder X-ray diffraction (XRD) patterns of (a) $\mathrm{CN}_{x}$ and $\mathrm{CN}_{\times} \mid \mathrm{Ni}_{2} \mathrm{P}(2 \mathrm{wt} \%)$ and (b) $\mathrm{Ni}_{2} \mathrm{P}$. 

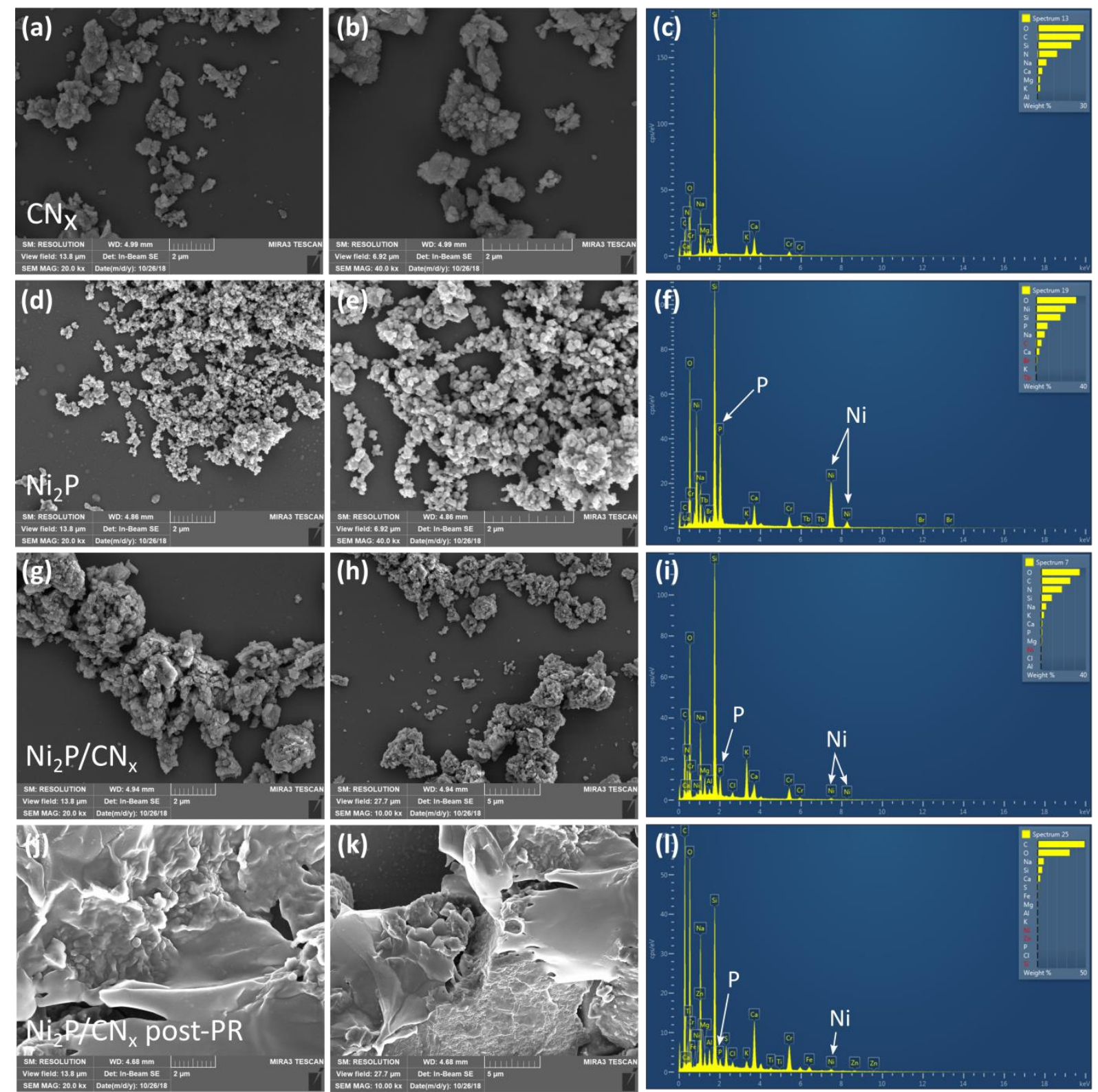

Figure S4. Scanning electron microscopy (SEM) images and energy dispersive X-ray spectroscopy (EDX) spectra of (a-c) $\mathrm{CN}_{x}$, (d-f) $\mathrm{Ni}_{2} \mathrm{P},(\mathbf{g}-\mathbf{i}) \mathrm{CN}_{x} \mid \mathrm{Ni}_{2} \mathrm{P}$ (2 wt\%), and (j-l) $\mathrm{CN}_{x} \mid \mathrm{Ni}_{2} \mathrm{P}$ (2 wt\%) after photoreforming. Samples were sputtered with $10 \mathrm{~nm}$ of Cr prior to imaging. Photoreforming conditions: $\mathrm{CN}_{\times} \mid \mathrm{Ni}_{2} \mathrm{P}(3.2 \mathrm{mg}$ ), PLA (50 mg), $1 \mathrm{M}$ aqueous $\mathrm{KOH}(2 \mathrm{~mL}$ ), simulated solar irradiation (AM 1.5G, 100 $\left.\mathrm{mW} \mathrm{cm}{ }^{-2}, 25^{\circ} \mathrm{C}, 50 \mathrm{~h}\right)$. 


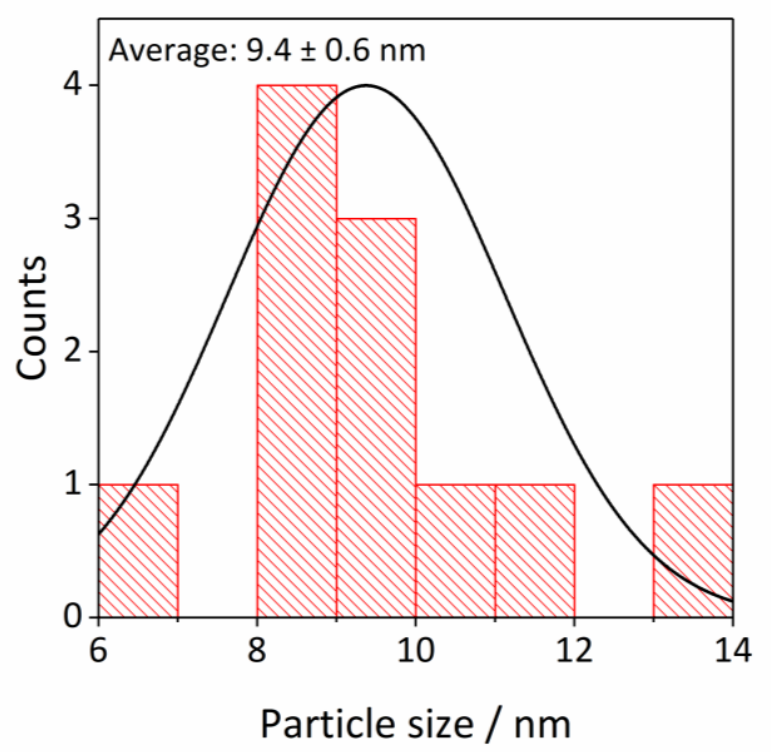

Figure S5. Particle size analysis of $\mathrm{Ni}_{2} \mathrm{P}$ nanoparticles annealed with $\mathrm{CN}_{\mathrm{x}}$, as measured from transmission electron microscopy (TEM) images.
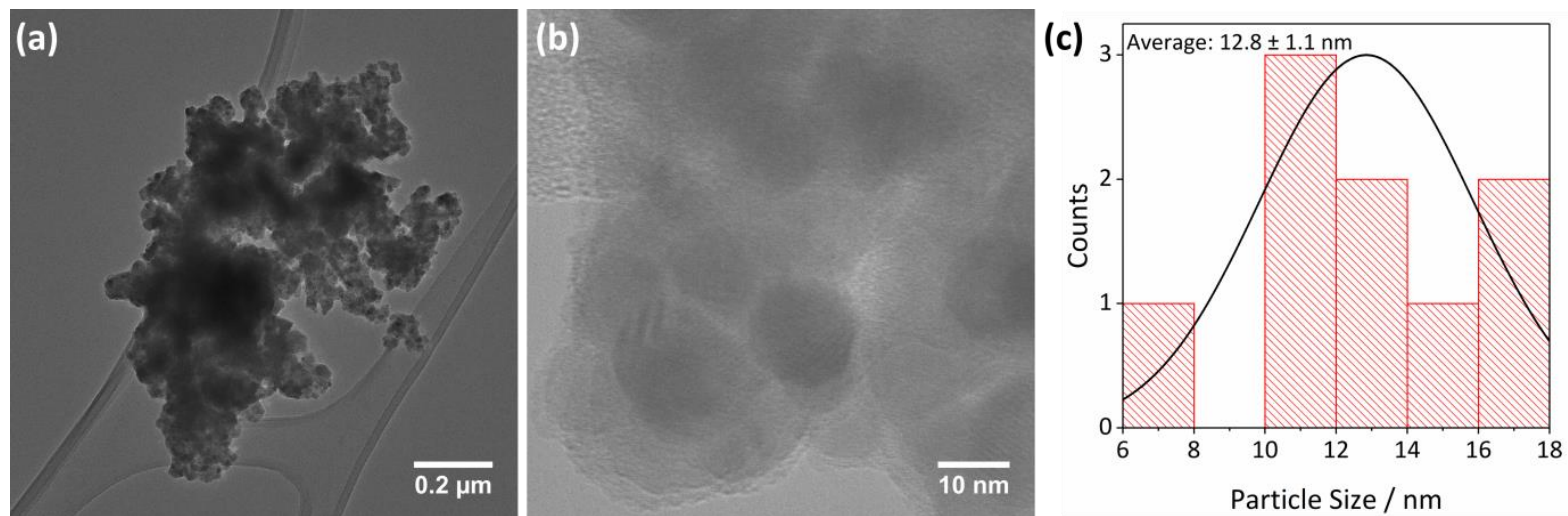

Figure S6. (a-b) TEM images of $\mathrm{Ni}_{2} \mathrm{P}$ nanoparticles. (c) Particle size analysis of the $\mathrm{Ni}_{2} \mathrm{P}$ nanoparticles. 

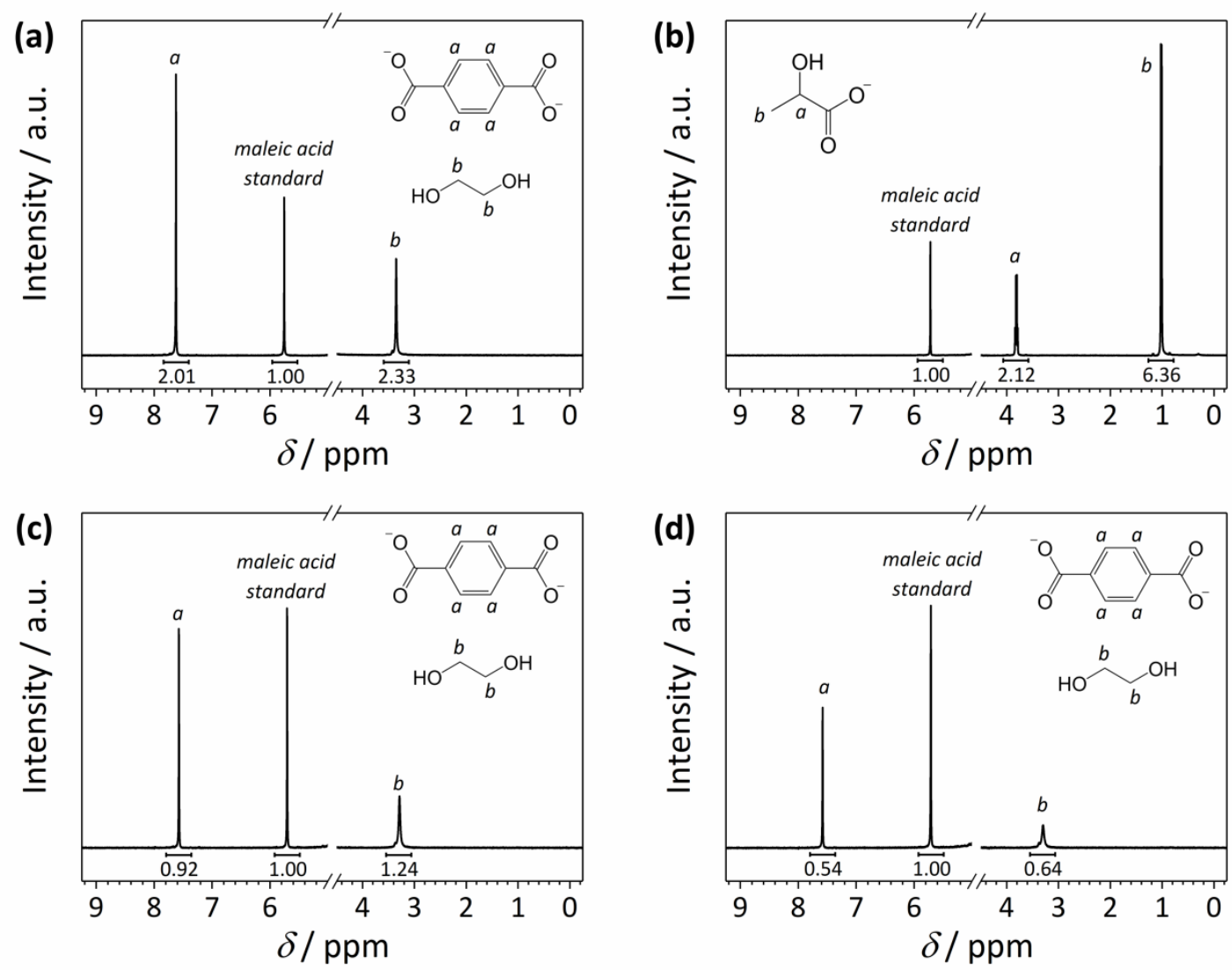

Figure S7. ${ }^{1} \mathrm{H}-\mathrm{NMR}$ quantification of polymer solubilisation after pre-treatment. (a) PET, (b) PLA, (c) PET bottle and (d) polyester microfiber in $1 \mathrm{M} \mathrm{NaOD}$ in $\mathrm{D}_{2} \mathrm{O}$ with maleic acid as a standard. 
(a)

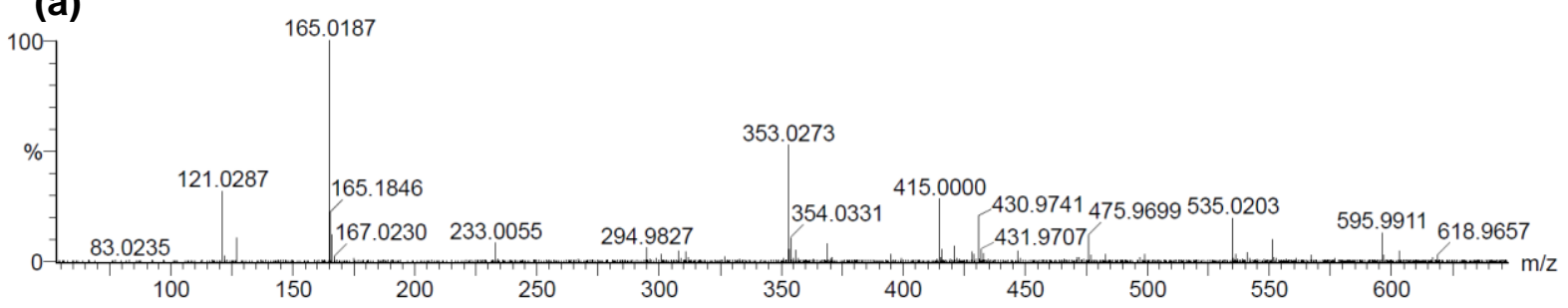

(b)

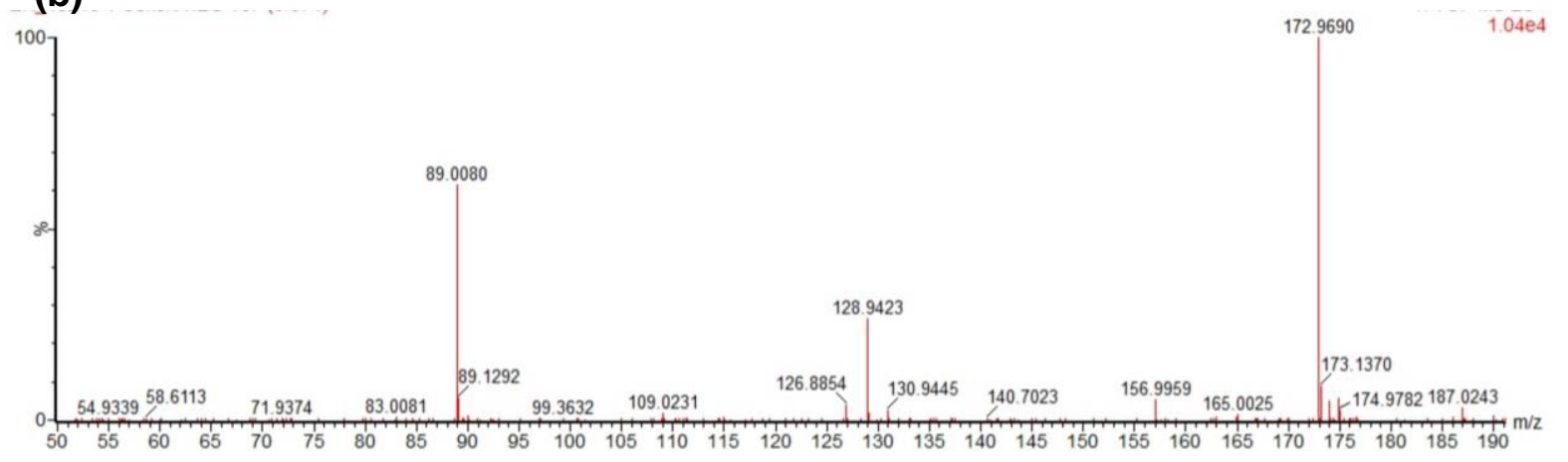

Figure S8. Liquid chromatography-mass spectrometry (LC-MS, negative ion mode) of solubilized (a) PET and (b) PLA components after pre-treatment. Samples were hydrolysed in $1 \mathrm{M}$ aq. $\mathrm{KOH}$ and then diluted with methanol to $0.01 \mathrm{M} \mathrm{KOH}$ for analysis, with measurements recorded up to $1000 \mathrm{~m} / \mathrm{z}$. In (a), The peak at $165 \mathrm{~m} / \mathrm{z}$ corresponds to terephthalate and the peak at $121 \mathrm{~m} / \mathrm{z}$ is a fragment of terephthalate (benzoate); no peaks are observed for common PET hydrolysis products such as mono(2hydroxyethyl) terephthalate (MHET, $210 \mathrm{~g} \mathrm{~mol}^{-1}$ ) or bis(2-hydroxyethyl) terephthalate (BHET, $254 \mathrm{~g}$ $\mathrm{mol}^{-1}$ ), but peaks at $121,353,451$, etc. $\mathrm{m} / \mathrm{z}$ may be from oligomer fragments. In (b), the peak at $89 \mathrm{~m} / \mathrm{z}$ corresponds to lactate, while the peaks at 129 and $173 \mathrm{~m} / \mathrm{z}$ are likely oligomer fragments. 

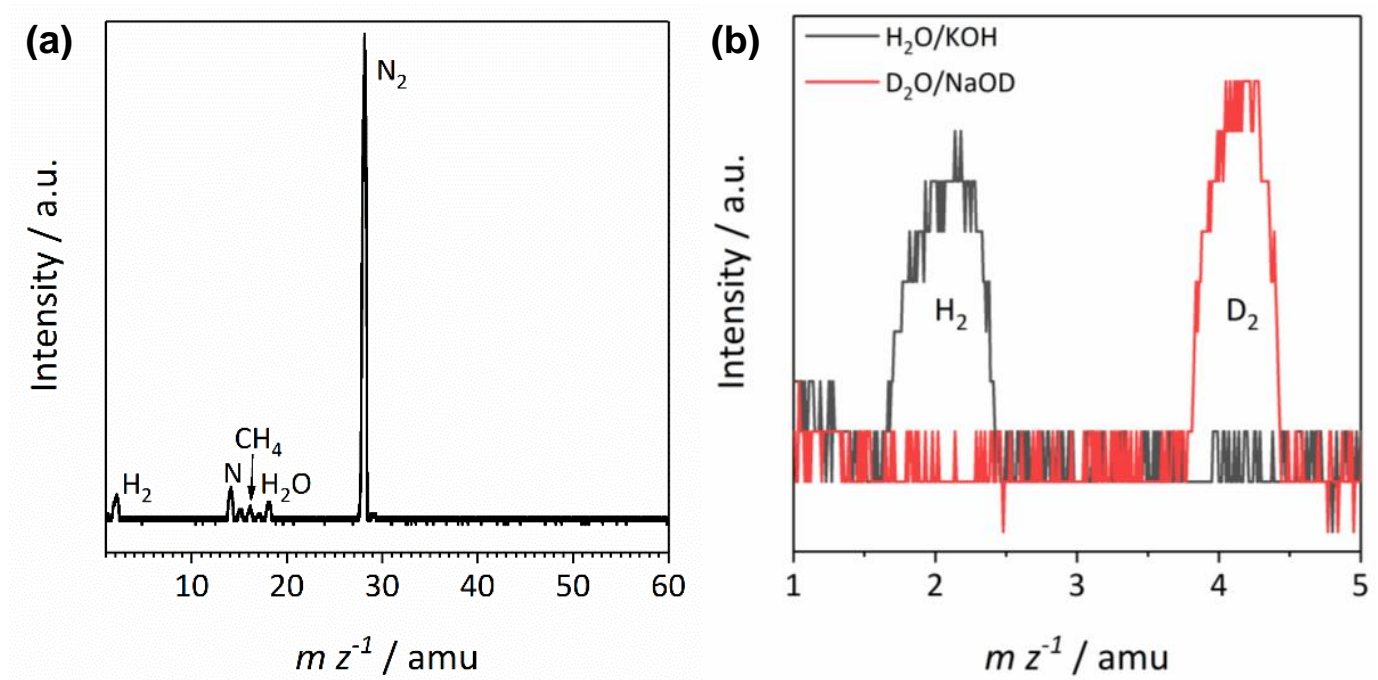

Figure S9. Mass spectra of the gas evolved after photoreforming (AM 1.5G, $100 \mathrm{~mW} \mathrm{~cm}^{-2}, 72 \mathrm{~h}$ ) of PET (25 mg mL ${ }^{-1}$ ) over $\mathrm{CN}_{\mathrm{x}} \mid \mathrm{Ni}_{2} \mathrm{P}(3.2 \mathrm{mg}$ ) in (a) $1 \mathrm{M}$ aq. $\mathrm{KOH}$ and (b) $1 \mathrm{M}$ aq. $\mathrm{KOH}$ or $1 \mathrm{M} \mathrm{NaOD}$ in $\mathrm{D}_{2} \mathrm{O}(2 \mathrm{~mL})$.

(a)

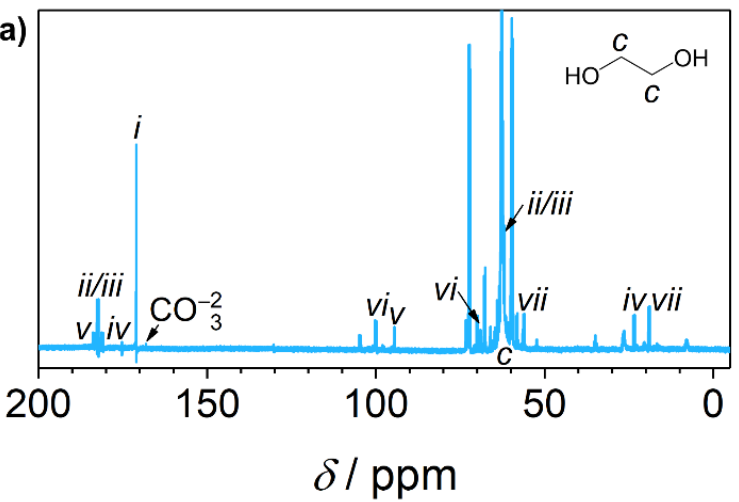

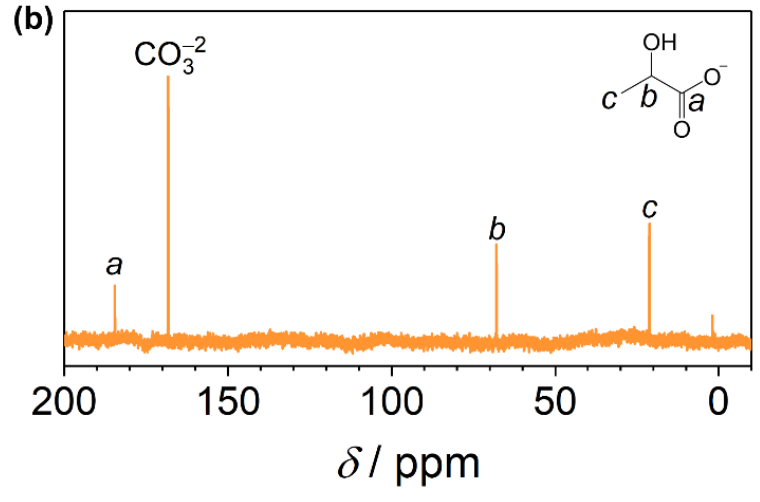

Figure S10. ${ }^{13} \mathrm{C}$-NMR spectrum of (a) ${ }^{13} \mathrm{C}$-labelled ethylene glycol (100 mg) and (b) PLA (3 mg) after photoreforming (AM 1.5G, $100 \mathrm{~mW} \mathrm{~cm}{ }^{-2}, 25^{\circ} \mathrm{C}, 5$ days) over $\mathrm{CN}_{\times} \mid \mathrm{Ni}_{2} \mathrm{P} 2 \mathrm{wt} \%$ (3.2 mg) in $1 \mathrm{M} \mathrm{NaOD}$ in $\mathrm{D}_{2} \mathrm{O}(2 \mathrm{~mL})$. The labels are attributed as follows: (i) formate, (ii) glyoxal, (iii) glycolate, (iv) acetate, (v) glyoxylate, (vi) glycoaldehyde, (vii) ethanol. 

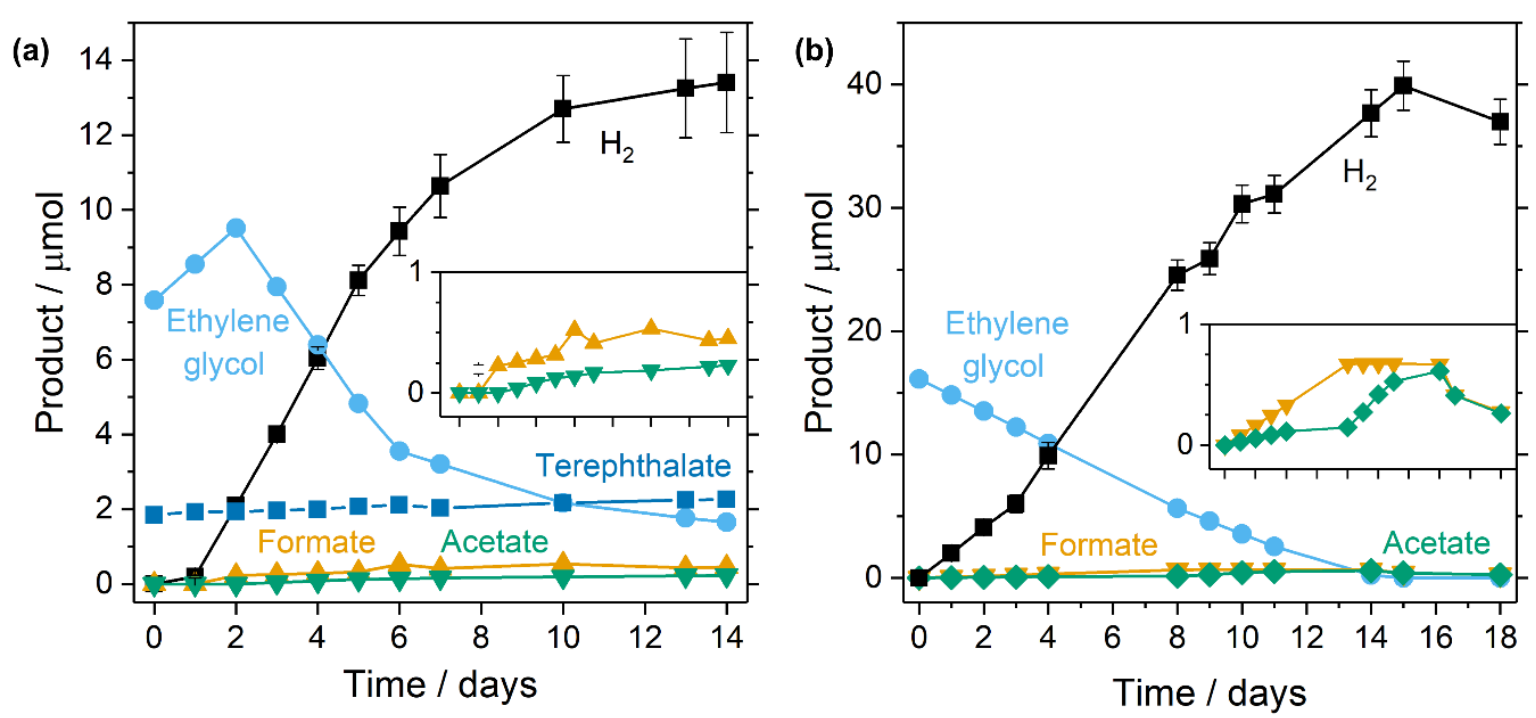

Figure S11. Long-term photoreforming of (a) PET (3 mg) and (b) ethylene glycol (1 mg) over $\mathrm{CN}_{x} \mid \mathrm{Ni}_{2} \mathrm{P}$ (1.6 mg mL ${ }^{-1}$ ). Conditions: $5 \mathrm{M}$ aq. $\mathrm{KOH}$ or $5 \mathrm{M} \mathrm{NaOD}$ in $\mathrm{D}_{2} \mathrm{O}$ (2 mL), simulated sunlight (AM 1.5G, 100 $\left.\mathrm{mW} \mathrm{cm}{ }^{-2}, 25^{\circ} \mathrm{C}\right)$. Aqueous products were analyzed using ${ }^{1} \mathrm{H}-\mathrm{NMR}$ with maleic acid as an internal standard. Insets show a zoomed-in view of the formate and acetate curves. The observed mass imbalance is due to unidentified oxidation products.

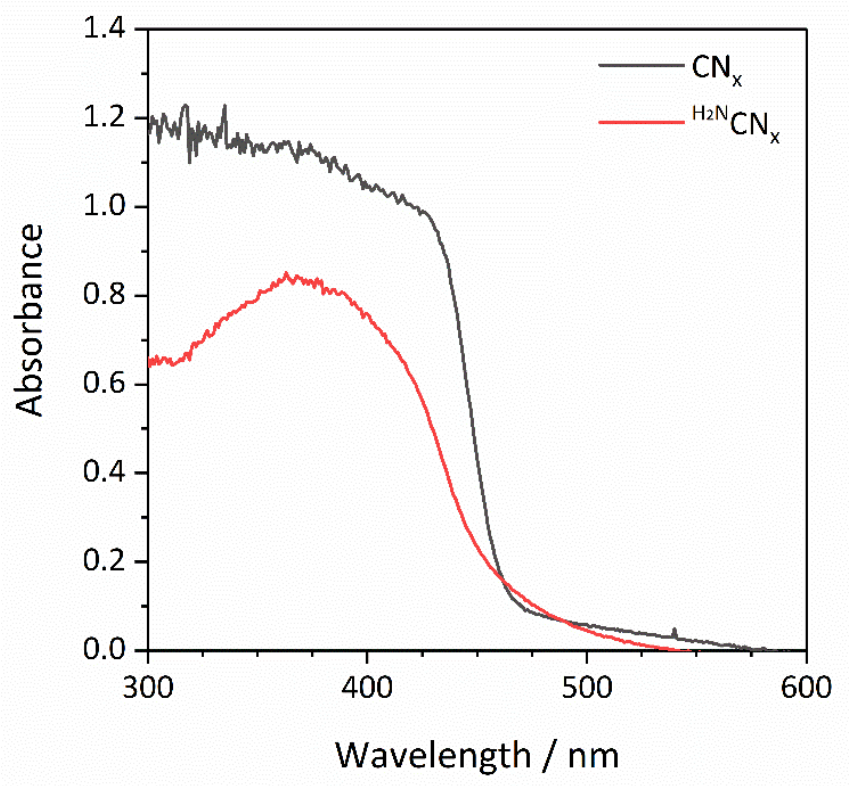

Figure S12. Comparison of the diffuse reflectance UV-Vis spectra of $\mathrm{CN}_{\mathrm{x}}$ and ${ }^{\mathrm{H} 2 \mathrm{~N}} \mathrm{CN}_{\mathrm{x}}$. 

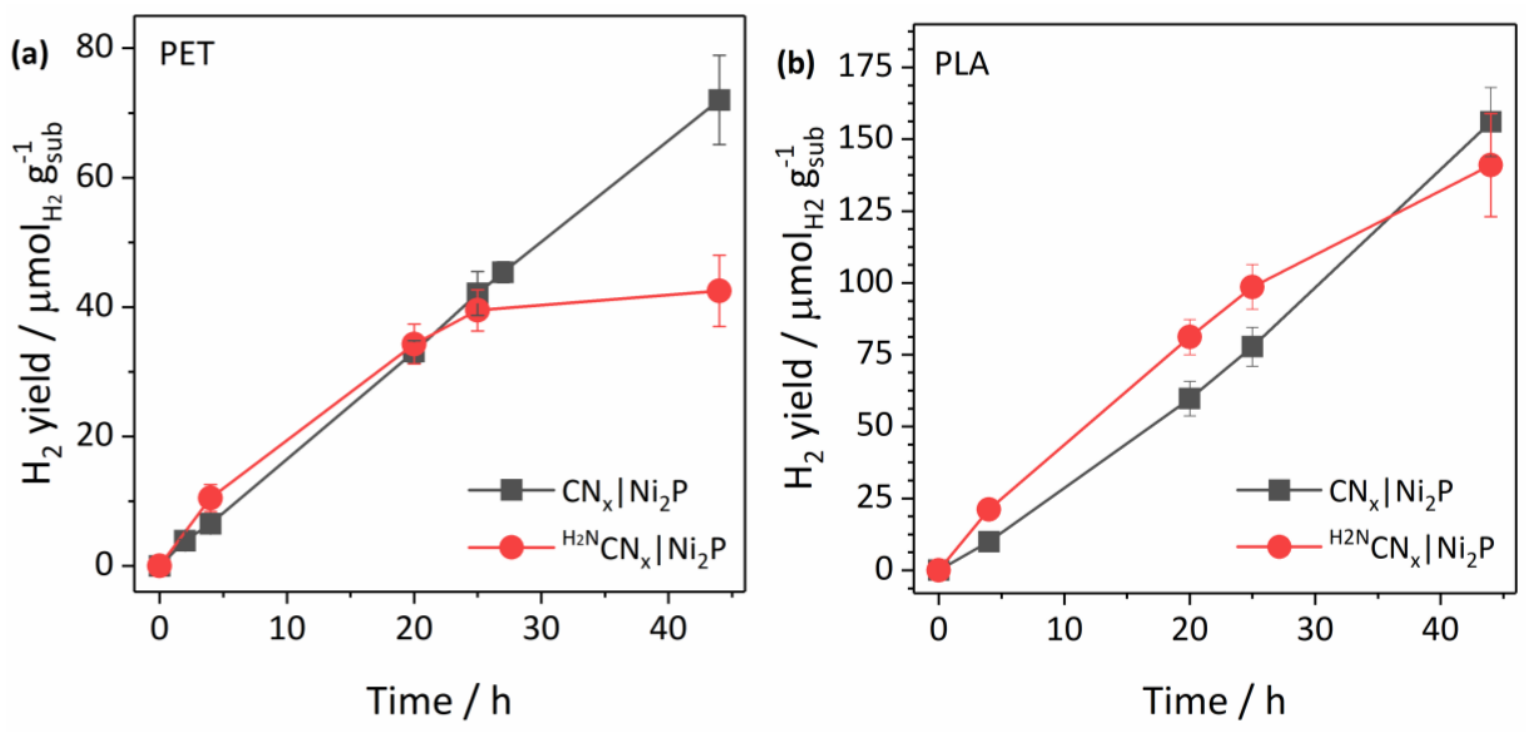

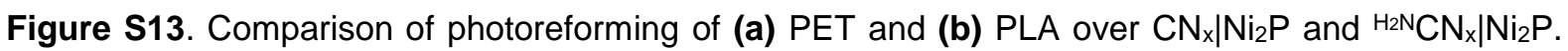
Conditions: photocatalyst $(3.2 \mathrm{mg})$, pre-treated polymer $\left(25 \mathrm{mg} \mathrm{mL}^{-1}\right)$, aqueous $\mathrm{KOH}(1 \mathrm{M}, 2 \mathrm{~mL})$, irradiation (AM 1.5G, $100 \mathrm{~mW} \mathrm{~cm}^{-2}, 25^{\circ} \mathrm{C}$ ). 

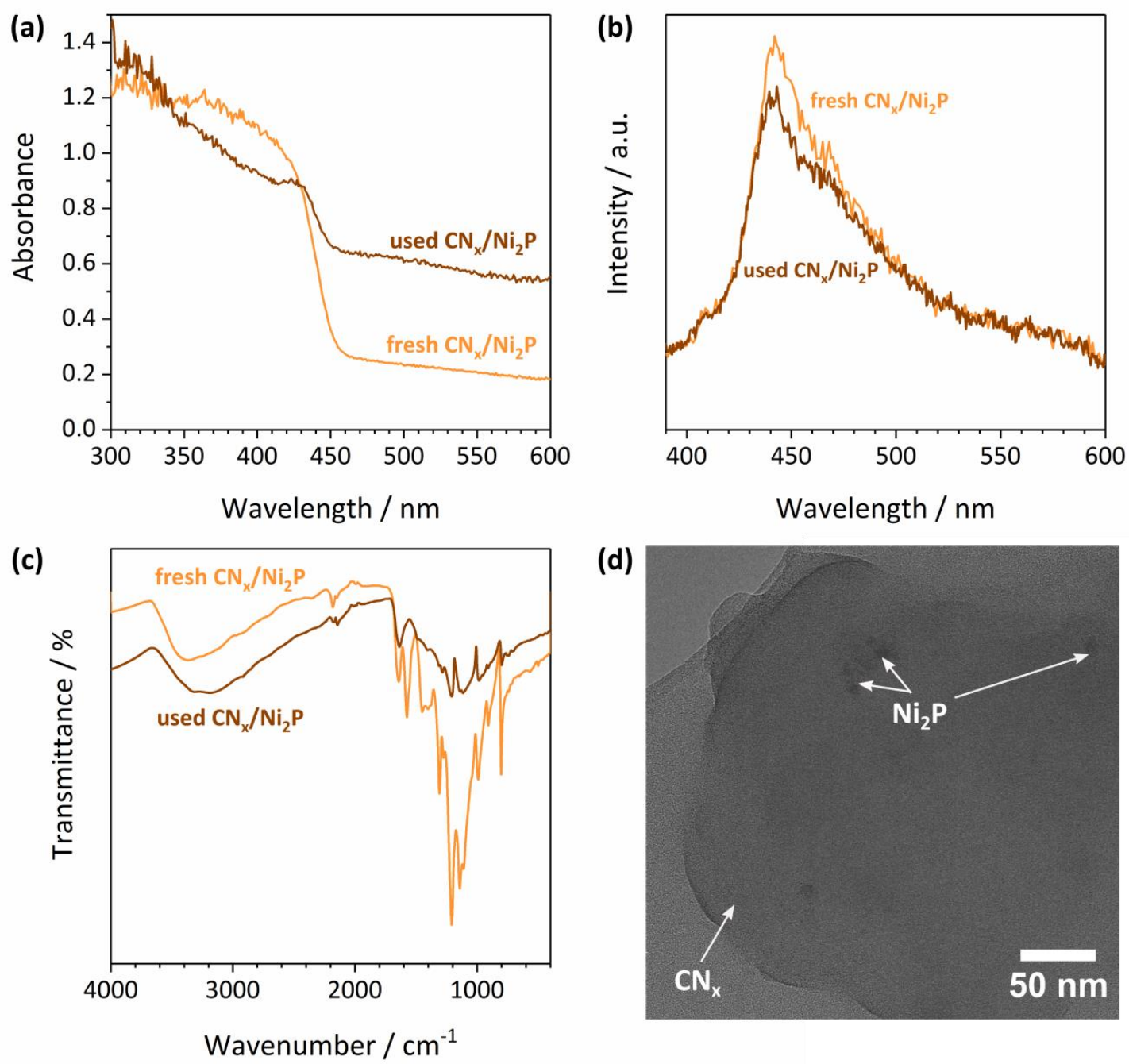

Figure S14. Post-photoreforming characterization of the $\mathrm{CN}_{x} \mid \mathrm{Ni}_{2} \mathrm{P}$ (2 wt\%) photocatalyst. (a) UV-Vis, (b) emission ( $\lambda_{\mathrm{ex}}=360 \mathrm{~nm}, \lambda_{\mathrm{em}}=450 \mathrm{~nm}$ ), and (c) FTIR spectra. (d) TEM image. Photoreforming conditions: $\mathrm{CN}_{\mathrm{x}} \mid \mathrm{Ni}_{2} \mathrm{P}$ (3.2 mg), PLA (50 mg), $1 \mathrm{M}$ aqueous $\mathrm{KOH}(2 \mathrm{~mL}$ ), simulated solar irradiation (AM $\left.1.5 \mathrm{G}, 100 \mathrm{~mW} \mathrm{~cm}^{-2}, 25^{\circ} \mathrm{C}, 50 \mathrm{~h}\right)$. 
(a)
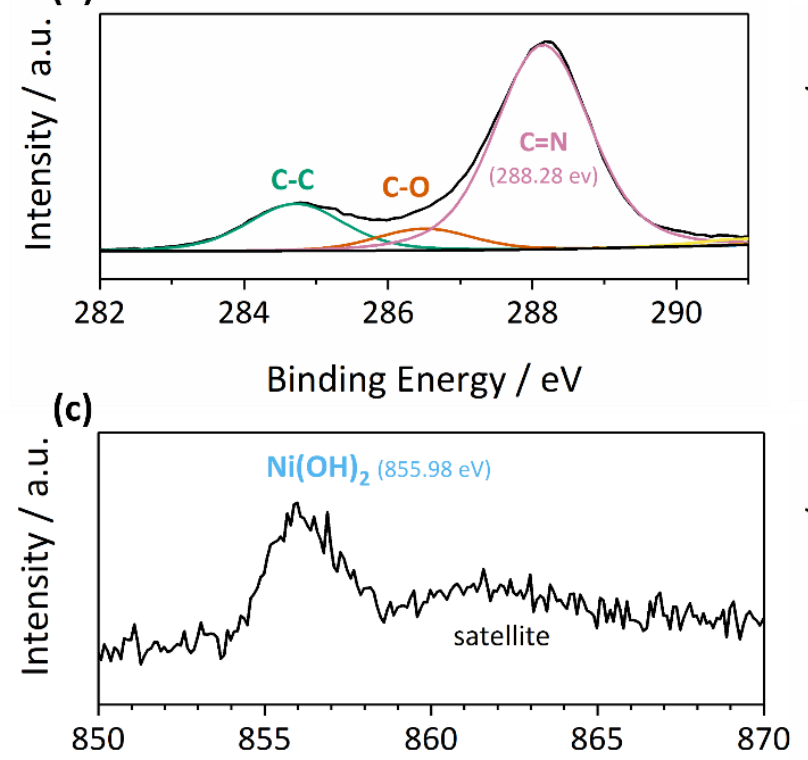

Binding Energy / eV (b)

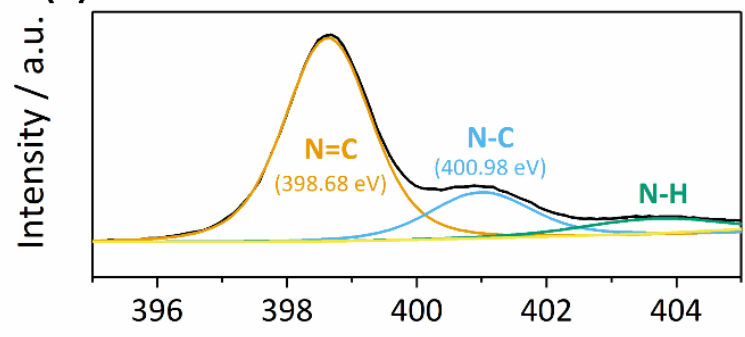

(d)

Binding Energy / eV

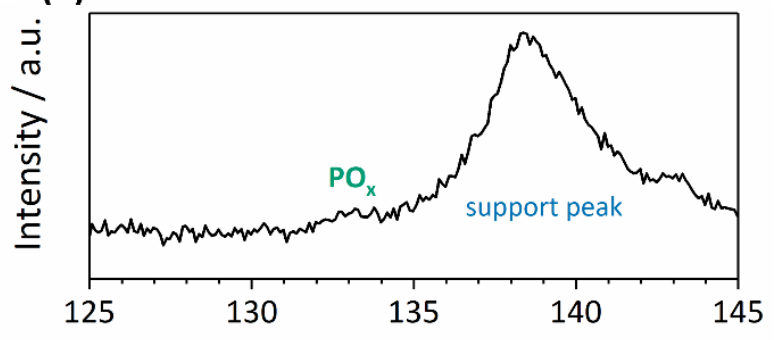

Binding Energy / eV

Figure S15. XPS spectra of the (a) $\mathrm{C}_{1 \mathrm{~s}}$, (b) $\mathrm{N}_{1 s}$, (c) $\mathrm{Ni}_{2 p}$, and (d) $\mathrm{P}_{2 p}$ edges of used $\mathrm{CN}_{\mathrm{x}} \mid \mathrm{Ni}_{2} \mathrm{P}$ (2 wt\%) after photoreforming. Photoreforming conditions: $\mathrm{CN}_{x} \mid \mathrm{Ni}_{2} \mathrm{P}$ (3.2 mg), PLA (50 mg), $1 \mathrm{M}$ aqueous $\mathrm{KOH}$ $\left(2 \mathrm{~mL}\right.$ ), simulated solar irradiation (AM 1.5G, $100 \mathrm{~mW} \mathrm{~cm}^{-2}, 25^{\circ} \mathrm{C}, 50 \mathrm{~h}$ ). 

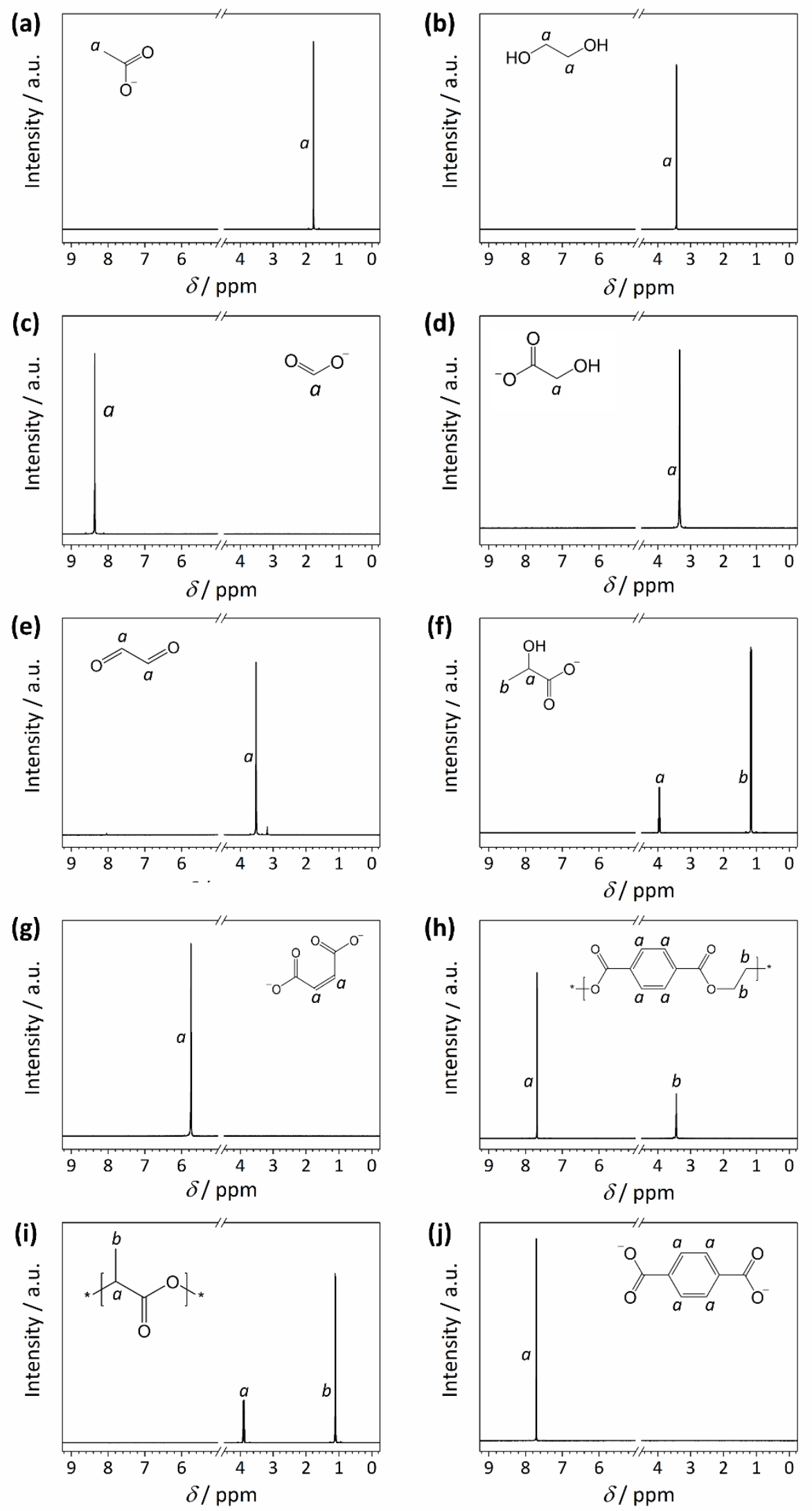

Figure S16. ' $\mathrm{H}-\mathrm{NMR}$ spectra of (a) acetate, (b) ethylene glycol, (c) formate, (d) glycolate, (e) glyoxal, (f) lactate, (g) maleate (used as a standard), (h) PET, (i) PLA, and (j) terephthalate in $1 \mathrm{M} \mathrm{NaOD}$ in $\mathrm{D}_{2} \mathrm{O}$. PET and PLA were pre-treated in $1 \mathrm{M} \mathrm{NaOD}$ in $\mathrm{D}_{2} \mathrm{O}$ for $24 \mathrm{~h}$ before data collection. 

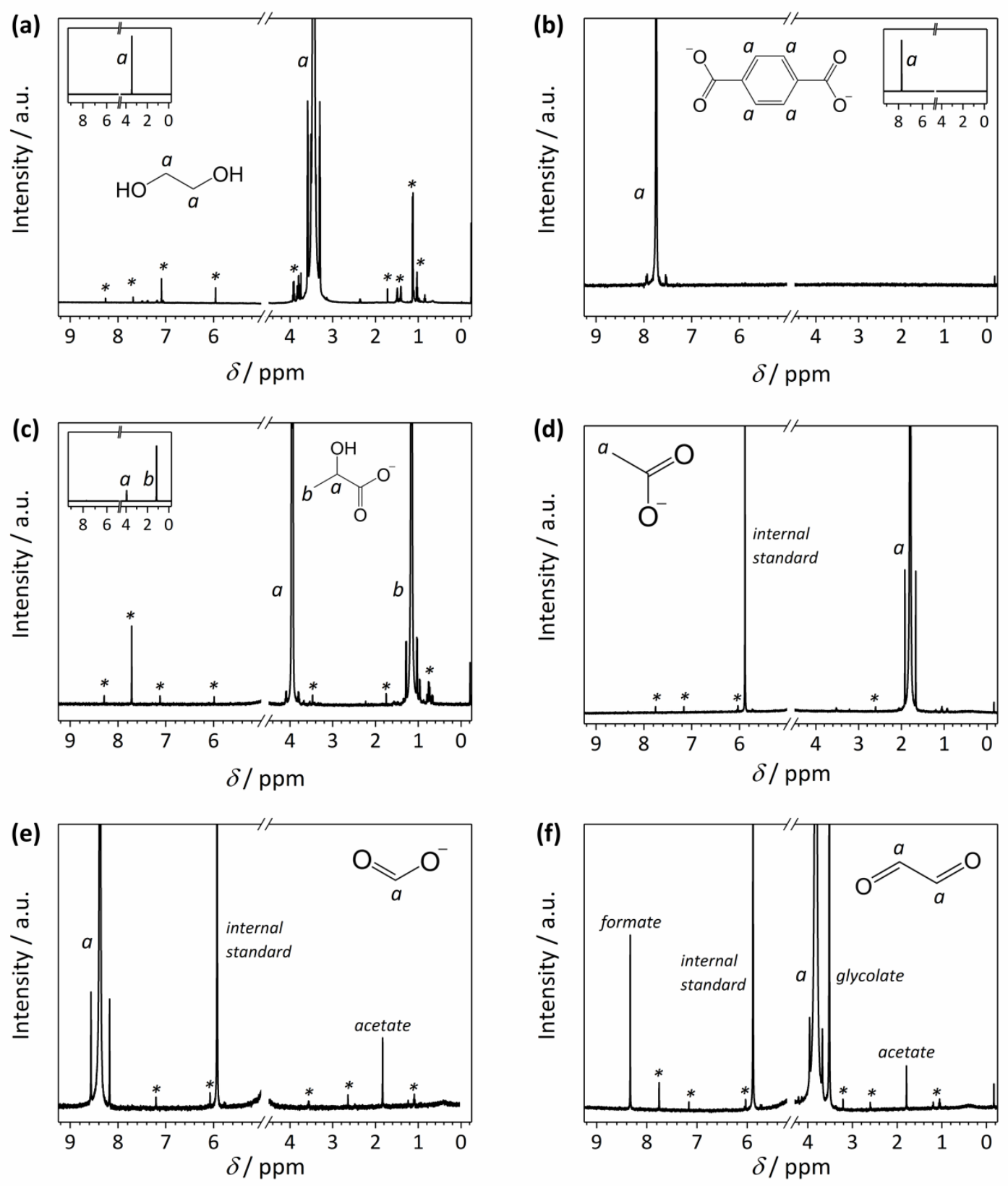

Figure S17. ${ }^{1} \mathrm{H}-\mathrm{NMR}$ spectra of (a) ethylene glycol, (b) terephthalate, and (c) lactate after 5 days simulated solar light irradiation. ${ }^{1} \mathrm{H}-\mathrm{NMR}$ spectra of (d) acetate, (e) formate and (f) glyoxal after $24 \mathrm{~h}$ simulated solar light irradiation. Maleic acid was used as an internal standard. Photoreforming conditions: $\mathrm{CN}_{\mathrm{x}} \mid \mathrm{Ni}_{2} \mathrm{P} 2 \mathrm{wt} \%$ (3.2 mg), $\mathrm{NaOD}(1 \mathrm{M})$ in $\mathrm{D}_{2} \mathrm{O}(2 \mathrm{~mL})$, substrate $\left(25 \mathrm{mg} \mathrm{mL}^{-1}\right)$, irradiation (AM 1.5G, $100 \mathrm{~mW} \mathrm{~cm}^{-2}, 25^{\circ} \mathrm{C}$ ). 
(a)
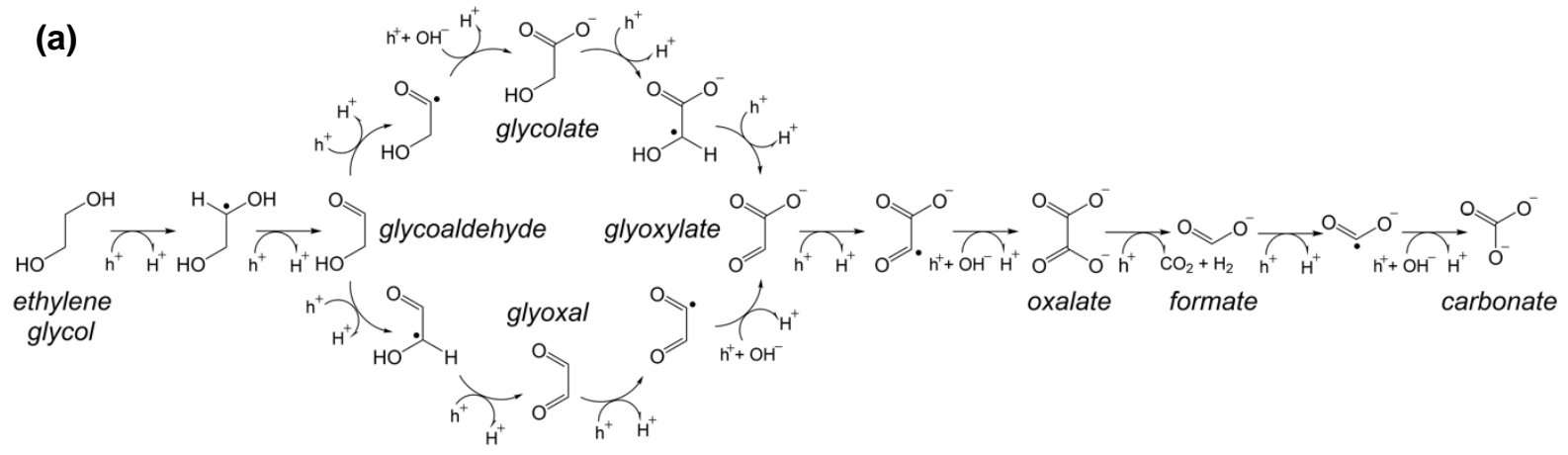

(b)

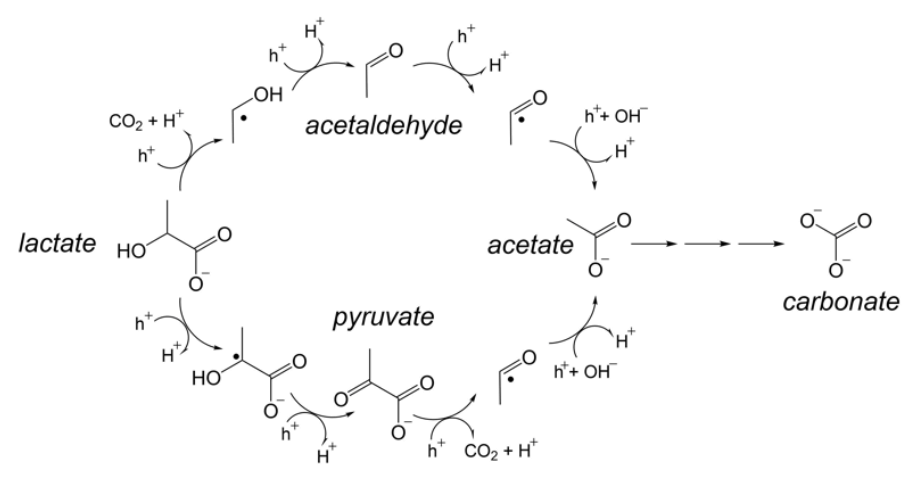

Figure S18. Proposed reaction scheme for the photo-oxidation of (a) ethylene glycol and (b) lactate. The mechanism is adapted from [14] and based on ${ }^{1} \mathrm{H}-\mathrm{NMR}$ analysis and comparison to literature. ${ }^{15-19}$

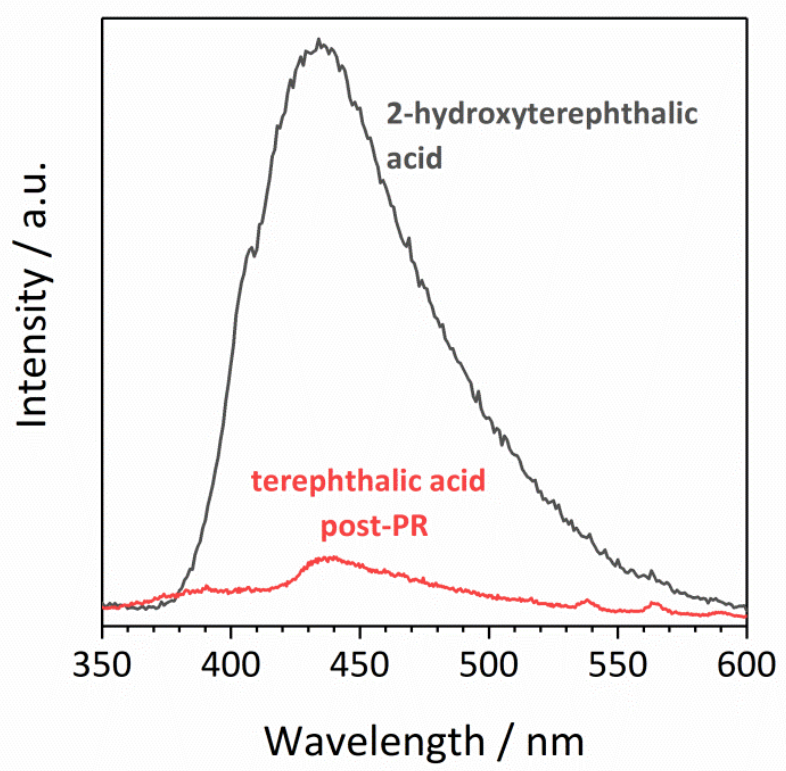

Figure S19. Emission spectra ( $\lambda_{\mathrm{ex}}=315 \mathrm{~nm}, \lambda_{\mathrm{em}}=430 \mathrm{~nm}$ ) of pure 2-hydroxyterephthalic acid in $1 \mathrm{M}$ aqueous $\mathrm{KOH}$ and terephthalic acid (50 mg) after photoreforming (AM 1.5G, $100 \mathrm{~mW} \mathrm{~cm}{ }^{-2}, 25^{\circ} \mathrm{C}, 20$ h) with $\mathrm{CN}_{x} \mid \mathrm{Ni}_{2} \mathrm{P}(3.2 \mathrm{mg})$ in $1 \mathrm{M}$ aqueous $\mathrm{KOH}(2 \mathrm{~mL})$. Terephthalic acid does not exhibit the characteristic $\lambda_{\mathrm{em}}=430 \mathrm{~nm}$ of the $\mathrm{OH}$ scavenger 2-hydroxyterephthalic acid, indicating that $\mathrm{OH}$ does not play a major role in the photoreforming mechanism. 

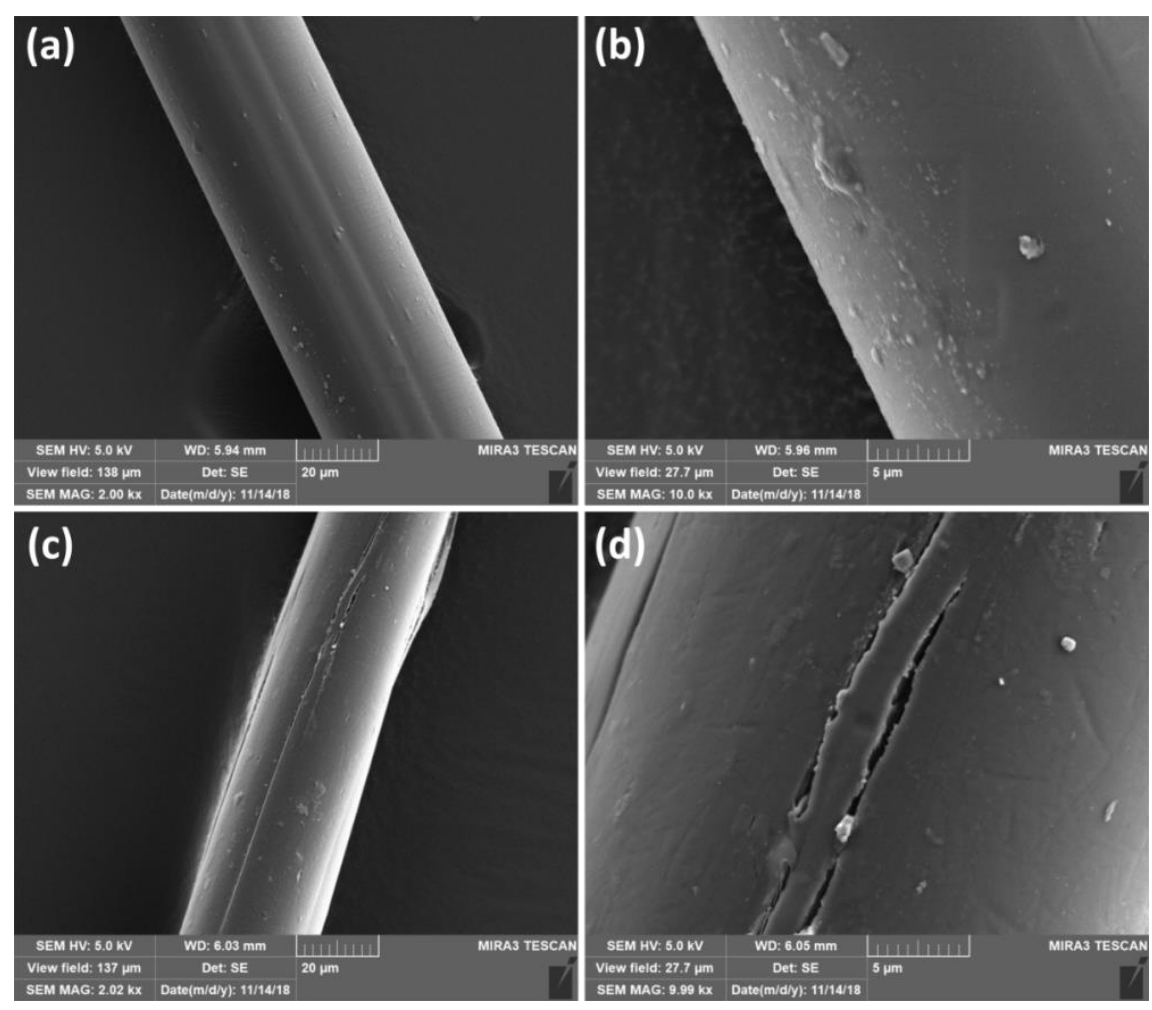

Figure S20. SEM images of a polyester microfiber (a-b) before photoreforming and (c-d) after photoreforming. Photoreforming conditions: $\mathrm{CN}_{\times} \mid \mathrm{Ni}_{2} \mathrm{P}(3.2 \mathrm{mg}), 1 \mathrm{M} \mathrm{KOH}(2 \mathrm{~mL})$, microfibers $(10 \mathrm{mg})$, simulated solar irradiation (AM 1.5G, $100 \mathrm{~mW} \mathrm{~cm}^{-2}, 25^{\circ} \mathrm{C}, 24 \mathrm{~h}$ ). Reformed microfibers were washed with $\mathrm{H}_{2} \mathrm{O}$ and dried under a stream of $\mathrm{N}_{2}$. Both samples were sputter-coated with $\mathrm{Pt}(10 \mathrm{~nm})$ prior to imaging.
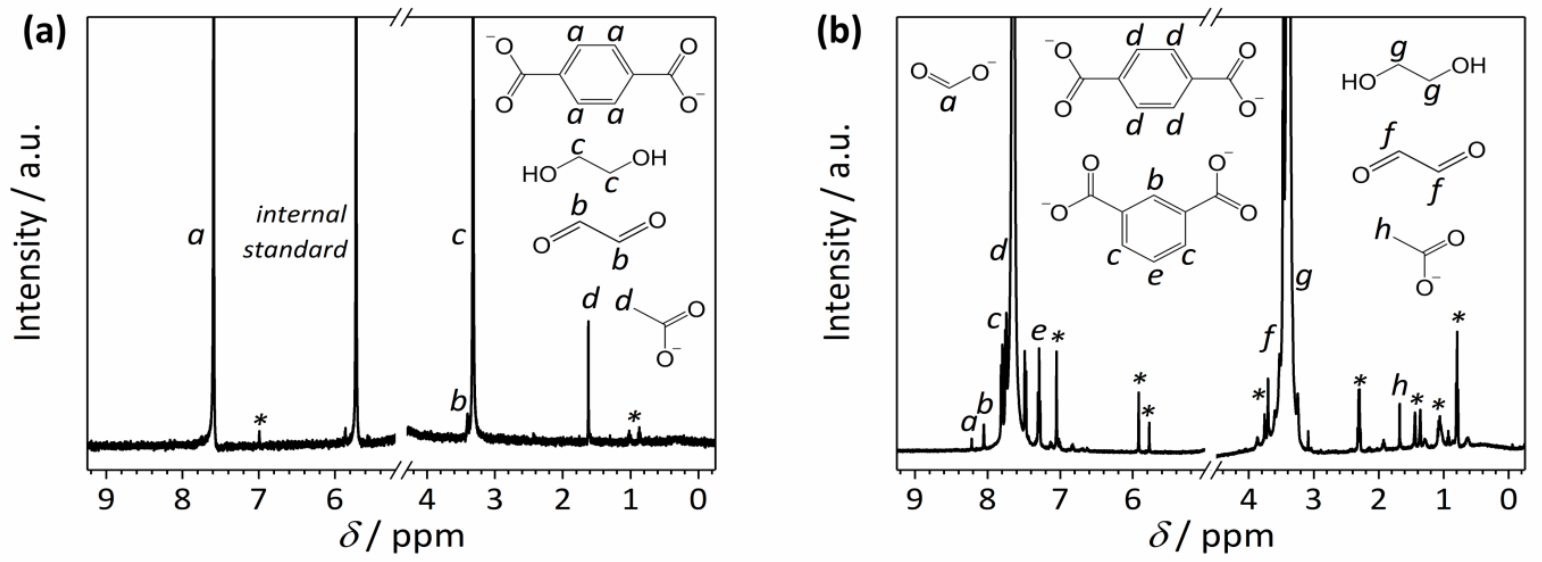

Figure S21. ${ }^{\mathrm{H}} \mathrm{H}-\mathrm{NMR}$ spectra of (a) polyester microfibers and (b) a PET water bottle after photoreforming. Photoreforming conditions: $\mathrm{CN}_{x} \mid \mathrm{Ni}_{2} \mathrm{P}(3.2 \mathrm{mg}), 1 \mathrm{M} \mathrm{NaOD}$ in $\mathrm{D}_{2} \mathrm{O}(2 \mathrm{~mL})$, pre-treated polymer (10 mg microfibers or $50 \mathrm{mg}$ PET bottle), simulated solar irradiation (AM 1.5G, $100 \mathrm{~mW} \mathrm{~cm} \mathrm{c}^{-2}$, $\left.25^{\circ} \mathrm{C}, 24 \mathrm{~h}\right)$. 


\section{References}

(1) Kawahara, Y.; Yoshioka, T.; Takarada, W.; Kikutani, T.; Tsuji, M. Alkaline Hydrolysis Kinetics of Poly (Ethylene Terephthalate) Fibers. J. Fiber Sci. Technol. 2016, 72, 9-16.

(2) NIST Chemistry WebBook. 1,2-Ethanediol https://webbook.nist.gov/cgi/cbook.cgi?ID=C107211\&Mask=2 (accessed Dec 4, 2018).

(3) Diane Sylvie Chauliac. Development of a Thermochemical Process for Hydrolysis of Polylactic Acid Polymers to L-Lactic Acid and Its Purification Using an Engineered Microbe, University of Florida, 2013.

(4) Emel'yanenko, V. N.; Verevkin, S. P.; Schick, C.; Stepurko, E. N.; Roganov, G. N.; Georgieva, M. K. The Thermodynamic Properties of S-Lactic Acid. Russ. J. Phys. Chem. A 2010, 84, 1491-1497.

(5) Indra, A.; Acharjya, A.; Menezes, P. W.; Merschjann, C.; Hollmann, D.; Schwarze, M.; Aktas, M.; Friedrich, A.; Lochbrunner, S.; Thomas, A.; et al. Boosting Visible-Light-Driven Photocatalytic Hydrogen Evolution with an Integrated Nickel Phosphide-Carbon Nitride System. Angew. Chem. Int. Ed. 2017, 56, 1653-1657.

(6) Lu, Z.; Li, C.; Han, J.; Wang, L.; Wang, S.; Ni, L.; Wang, Y. Construction OD/2D Heterojunction by Highly Dispersed $\mathrm{Ni}_{2} \mathrm{P}$ QDs Loaded on the Ultrathin $\mathrm{g}-\mathrm{C}_{3} \mathrm{~N}_{4}$ Surface towards Superhigh Photocatalytic and Photoelectric Performance. Appl. Catal. B Environ. 2018, 237, 919-926.

(7) Ye, P.; Liu, X.; locozzia, J.; Yuan, Y.; Gu, L.; Xu, G.; Lin, Z. A Highly Stable Non-Noble Metal Ni2P CoCatalyst for Increased $\mathrm{H}_{2}$ Generation by $\mathrm{g}-\mathrm{C}_{3} \mathrm{~N}_{4}$ under Visible Light Irradiation. J. Mater. Chem. A 2017, 5, 8493-8498.

(8) Zeng, D.; Xu, W.; Ong, W.-J.; Xu, J.; Ren, H.; Chen, Y.; Zheng, H.; Peng, D.-L. Toward Noble-Metal-Free Visible-Light-Driven Photocatalytic Hydrogen Evolution: Monodisperse Sub-15 Nm Ni $2 \mathrm{P}$ Nanoparticles Anchored on Porous g- $\mathrm{C}_{3} \mathrm{~N}_{4}$ Nanosheets to Engineer 0D-2D Heterojunction Interfaces. Appl. Catal. B Environ. 2018, 221, 47-55.

(9) Zhao, H.; Sun, S.; Jiang, P.; Xu, Z. J. Graphitic $\mathrm{C}_{3} \mathrm{~N}_{4}$ Modified by Ni2P Cocatalyst: An Efficient, Robust and Low Cost Photocatalyst for Visible-Light-Driven $\mathrm{H}_{2}$ Evolution from Water. Chem. Eng. J. 2017, 315 , 296-303.

(10) Wen, J.; Xie, J.; Shen, R.; Li, X.; Luo, X.; Zhang, H.; Zhang, A.; Bi, G. Markedly Enhanced Visible-Light Photocatalytic $\mathrm{H}_{2}$ Generation over $\mathrm{g}-\mathrm{C}_{3} \mathrm{~N}_{4}$ Nanosheets Decorated by Robust Nickel Phosphide $\left(\mathrm{Ni}_{12} \mathrm{P}_{5}\right)$ Cocatalysts. Dalt. Trans. 2017, 46, 1794-1802.

(11) Yu, J.; Wang, S.; Cheng, B.; Lin, Z.; Huang, F. Noble Metal-Free $\mathrm{Ni}(\mathrm{OH})_{2}-\mathrm{g}-\mathrm{C}_{3} \mathrm{~N}_{4}$ Composite Photocatalyst with Enhanced Visible-Light Photocatalytic $\mathrm{H}_{2}-$ Production Activity. Catal. Sci. Technol. 2013, 3, 1782.

(12) Kawai, T.; Sakata, T. Photocatalytic Hydrogen Production from Water by the Decomposition of PolyVinylchloride, Protein, Algae, Dead Insects, and Excrement. Chem. Lett. 1981, 81-84.

(13) Uekert, T.; Kuehnel, M. F.; Wakerley, D. W.; Reisner, E. Plastic Waste as a Feedstock for Solar-Driven $\mathrm{H}_{2}$ Generation. Energy Environ. Sci. 2018, 11, 2853-2857.

(14) Puga, A. V. Photocatalytic Production of Hydrogen from Biomass-Derived Feedstocks. Coord. Chem. Rev. 2016, 315, 1-66.

(15) Yue, H.; Zhao, Y.; Ma, X.; Gong, J. Ethylene Glycol: Properties, Synthesis, and Applications. Chem. Soc. Rev. Chem. Soc. Rev 2012, 41, 4089-4380.

(16) Rossiter, W. J.; Brown, P. W.; Godette, M. The Determination of Acidic Degradation Products in Aqueous Ethylene Glycol and Propylene Glycol Solutions Using lon Chromatography. Sol. Energy Mater. 1983, 9, 267-279.

(17) Chauhan, N. L.; Dameera, V.; Chowdhury, A.; Juvekar, V. A.; Sarkar, A. Electrochemical Oxidation of Ethylene Glycol in a Channel Flow Reactor. Catal. Today 2018, 309, 126-132.

(18) Lomate, S.; Katryniok, B.; Dumeignil, F.; Paul, S. High Yield Lactic Acid Selective Oxidation into Acetic Acid over a Mo-V-Nb Mixed Oxide Catalyst. Sustain. Chem. Process. 2015, 3, 5.

(19) Harada, H.; Sakata, T.; Ueda, T. Effect of Semiconductor on Photocatalytic Decomposition of Lactic Acid. J. Am. Chem. Soc. 1985, 107, 1773-1774. 